\title{
Efficient Erasure Correcting Codes
}

\author{
Michael G. Luby, Michael Mitzenmacher, M. Amin Shokrollahi, and Daniel A. Spielman
}

\begin{abstract}
We introduce a simple erasure recovery algorithm for codes derived from cascades of sparse bipartite graphs and analyze the algorithm by analyzing a corresponding discrete-time random process. As a result, we obtain a simple criterion involving the fractions of nodes of different degrees on both sides of the graph which is necessary and sufficient for the decoding process to finish successfully with high probability. By carefully designing these graphs we can construct for any given rate $R$ and any given real number $\epsilon$ a family of linear codes of rate $R$ which can be encoded in time proportional to $\ln (1 / \epsilon)$ times their block length $n$. Furthermore, a codeword can be recovered with high probability from a portion of its entries of length $(1+\epsilon) R n$ or more. The recovery algorithm also runs in time proportional to $n \ln (1 / \epsilon)$. Our algorithms have been implemented and work well in practice; various implementation issues are discussed.
\end{abstract}

Index Terms-Erasure channel, large deviation analysis, lowdensity parity-check codes.

\section{INTRODUCTION}

A LINEAR error-correcting code of block length $n$ and dimension $k$ over a finite field $\mathbb{F}_{q}$-an $[n, k]_{q}$-code for short-is a $k$-dimensional linear subspace of the standard vector space $\mathbb{F}_{q}^{n}$. The elements of the code are called codewords. To the code $C$ there corresponds an encoding map Enc which is an isomorphism of the vector spaces $\mathbb{F}_{q}^{k}$ and $C$. A sender, who wishes to transmit a vector of $k$ elements in $\mathbb{F}_{q}$ to a receiver, uses the mapping Enc to encode that vector into a codeword. The rate $k / n$ of the code is a measure for the amount of real information in each codeword. The minimum distance of the

Manuscript received June 7, 1999; revised September 8, 2000. The work of M. G. Luby was supported in part by the National Science Foundation under Operating Grant NCR-9416101, and by the United States-Israel Binational Science Foundation under Grant 92-00226. The work of M. Mitzenmacher was supported in part by an Alfred P. Sloan Research Fellowship and by the National Science Foundation under CAREER Grant CCR-9983832. A substantial portion of this work was performed while M. Mitzenmacher was at the Computer Science Department, University of California, Berkeley, under NSF Grant CCR-9505448. The work of M. A. Shokrollahi was supported in part by a Habilitationsstipendium of the Deutsche Forschungsgemeinschaft under Grant Sh 57/1-1. This work was performed while D. A. Speilman was visiting the University of California, Berkeley, and supported by an NSF mathematical sciences Postdoctoral Fellowship.

M. G. Luby and M. A. Shokrollahi were with the International Computer Science Institute, Berkeley, CA USA. They are now with Digital Fountain, Inc, San Francisco, CA 94110 USA (e-mail: luby@digitalfountain.com; amin@digital.fountain.com).

M. Mitzenmacher was with Digital Equipment Corporation, Systems Research Center, Palo Alto, CA USA. He is now with Harvard University, Cambridge, MA 02138 USA (e-mail: michaelm@eecs.harvard.edu).

D. A. Spielman is with is with the Department of Mathematics, the Massachusetts Institute of Technology, Cambridge, MA 02139 (e-mail: spielman@math.mit.edu)

Communicated by F. R. Kschischang, Associate Editor for Coding Theory. Publisher Item Identifier S 0018-9448(01)00735-0. code is the minimum Hamming distance between two distinct codewords. A linear code of block length $n$, dimension $k$, and minimum distance $d$ over $\mathbb{F}_{q}$ is called an $[n, k, d]_{q}$-code.

Linear codes can be used to reliably transmit information from a sender to a receiver: the sender first encodes the desired word into a codeword and transmits the codeword over the transmission channel. Depending on the nature of the errors imposed on the codeword through the channel, the receiver then applies appropriate algorithms to decode the received word. In this paper, we assume that the receiver knows the position of each received symbol within the stream of all codeword symbols. We adopt as our model of errors the erasure channel, introduced by Elias [4], in which each codeword symbol is lost with a fixed constant probability $p$ in transit independent of all the other symbols. Elias [4] showed that the capacity of the erasure channel is $1-p$ and that a random linear code can be used to transmit over the erasure channel at any rate $R<1-p$.

It is easy to see that a code of minimum distance $d$ is capable of recovering $d-1$ or fewer erasures. Furthermore, a closer look reveals that this task can be done in time $O\left(n^{3}\right)$. The code is optimal with respect to recovering erasures if it can recover from any set of $k$ coordinates of the codeword, i.e., if $d-1=n-k$. Such codes are called minimum-distance separable (MDS) codes. A standard class of MDS codes is given by Reed-Solomon (RS) codes [16]. The connection of these codes with polynomial arithmetic allows for encoding and decoding in time $O\left(n \log ^{2} n \log \log n\right)$ (see, [3, Ch. 11.7] and [16, p. 369]). However, for small values of $n$, quadratic time algorithms are faster than the theoretically, asymptotically fast algorithms for the RS-based codes, and for larger values of $n$ the $O\left(\log ^{2} n \log \log n\right)$ multiplicative overhead in the running time of the fast algorithms (along with a moderate-sized constant hidden by the big-Oh notation) is large. Obviously, one cannot hope for better information recovery than that given by RS codes, but faster encoding and decoding times are desirable. In this paper, we design fast linear-time algorithms for transmitting just below channel capacity. For all $\epsilon>0$, we produce rate $R=1-p(1+\epsilon)$ codes along with decoding algorithms that recover from the random loss of a $p$ fraction of the transmitted symbols in time proportional to $n \ln (1 / \epsilon)$ with high probability, where $n$ is the block length. These codes can be encoded in time proportional to $n \ln (1 / \epsilon)$. The fastest previously known encoding and decoding algorithms [1] with such a performance guarantee have run times proportional to $n \ln (1 / \epsilon) / \epsilon$.

The overall structure of our codes is related to the low-density parity-check codes introduced by Gallager [6], which have been the subject of a great deal of recent work (see, for example, [10], [11], and [15]). We also use some ideas related to the codes introduced in [25] for error correction. Because we examine the erasure setting, however, our work includes several innovations, 
including a simple linear time decoding algorithm and the use of irregularity. We explain the general construction along with the encoding and decoding algorithms fully in Section II.

Our encoding and decoding algorithms are almost symmetrical. Both are very simple, computing exactly one exclusive-or operation for each edge in a randomly chosen bipartite graph. As in many similar applications, the graph is chosen to be sparse, which immediately implies that the encoding and decoding algorithms are fast. Unlike many similar applications, the graph is not regular; instead, it is quite irregular with a carefully chosen degree sequence. We describe the decoding algorithm as a process on the graph in Section II-B. Our main tool is a model that characterizes almost exactly the performance of the decoding algorithm as a function of the degree sequence of the graph. In Section III, we use this tool to model the progress of the decoding algorithm by a set of differential equations. The solution to these equations can then be expressed as polynomials in one variable with coefficients determined by the degree sequence. The positivity of one of these polynomials on the interval $(0,1]$ with respect to a parameter $\delta$ guarantees that, with high probability, the decoding algorithm can recover almost all the message symbols from a loss of up to a $\delta$ fraction of the codeword symbols (see Proposition 2). The complete success of the decoding algorithm can then be demonstrated by combinatorial arguments.

Our analytical tools allow us to almost exactly characterize the performance of the decoding algorithm for any given degree sequence. Furthermore, they also help us to design good irregular degree sequences. In Section IV, we describe, given a parameter $\epsilon>0$, a degree sequence for which the decoding is successful with high probability for an erasure fraction $\delta$ that is within $\epsilon$ of $1-R$. Although these graphs are irregular, with some nodes of degree $1 / \epsilon$, the average node degree is only $\ln (1 / \epsilon)$. This is one of the central results of the paper, i.e., a code with encoding and decoding times proportional to $n \ln (1 / \epsilon)$ that can recover from an erasure fraction that is within $\epsilon$ of optimal.

In Section V, we discuss issues concerning practical implementations of our algorithms. This section includes methods for finding good degree sequences based on linear programming, and timings of the implementations. In the last section, we summarize the main results of this paper, and discuss recent developments following the publication of a preliminary version [13].

\section{GRAPH CODES}

In this section we introduce a new class of codes. Special subclasses of these codes turn out to be almost MDS in the following sense: an $[n, k]_{q}$-code in this subclass is capable of recovering the message from a random set of $k(1+\epsilon)$ coordinate places with high probability, where $\epsilon$ is a small real number. A more precise statement is provided later in Section III. The advantages of these codes are that they have linear time encoding and decoding algorithms, and that the alphabet size $q$ can be arbitrary. For simplicity, in the following we assume that the symbols are bits, i.e., that $q=2$.

We explain the overall construction of the codes, as well as introduce simple and efficient encoding and recovery algorithms.

\section{A. Erasure Codes via Bipartite Graphs}

We define a code $\mathcal{C}(B)$ with $k$ message bits and $\beta k$ redundant bits, where $0<\beta<1$, by associating these bits with a bipartite graph $B .{ }^{1}$ Following standard terminology, we refer to the $\beta k$ redundant bits as check bits. The graph $B$ has $k$ left nodes and $\beta k$ right nodes, corresponding to the message bits and the check bits, respectively. Hence, in the following, we refer to the left nodes of a bipartite graph as its message bits and to the right nodes as its check bits.

The encoding of $\mathcal{C}(B)$ is determined by setting each check bit to be the $\oplus$ (XOR) of its neighboring message bits in $B$ (see Fig. 1(a)). Thus, the encoding time is proportional to the number of edges in $B$, and our codes are systematic. ${ }^{2}$

Our main contribution is the design and analysis of the bipartite graph $B$ so that the repetition of the following simplistic decoding operation recovers all the missing message bits.

\section{Algorithm 1 (Erasure Decoding) \\ Given the value of a check bit and all but one of the message bits on which it de- pends, set the missing message bit to be the xOR of the check bit and its known message bits.}

See Fig. 1(b) for an example of this algorithm, and Fig. 2 for an example of full recovery.

We introduce methods for the design of sparse random graphs where repetition of this operation recovers all the message bits with high probability if a random subset of $(1-\epsilon) \beta k$ of the message bits have been lost from $\mathcal{C}(B)$.

To produce codes that can correct erasures of check bits as well as message bits, we cascade codes of the form $\mathcal{C}(B)$ : we first use $\mathcal{C}(B)$ to produce $\beta k$ check bits for the original $k$ message bits, we then use a similar code to produce $\beta^{2} k$ check bits for the $\beta k$ check bits of $\mathcal{C}(B)$, and so on (see Fig. 3). At the last level, we may use a more conventional erasure correcting code (e.g., an RS code, if the alphabet size is large enough).

Formally, we construct a family of $\operatorname{codes} \mathcal{C}\left(B_{0}\right), \ldots, \mathcal{C}\left(B_{m}\right)$ from a family of graphs $B_{0}, \ldots, B_{m}$, where $B_{i}$ has $\beta^{i} k$ left nodes and $\beta^{i+1} k$ right nodes. We select $m$ so that $\beta^{m+1} k$ is roughly $\sqrt{k}$. We end the cascade with an erasure correcting code $C$ of rate $1-\beta$ with $\beta^{m+1} k$ message bits for which we know how to recover from the random loss of $\beta$ fraction of its bits with high probability. We then define the code $\mathcal{C}\left(B_{0}, B_{1}, \ldots, B_{m}, C\right)$ to be a code with $k$ message bits and

$$
\sum_{i=1}^{m+1} \beta^{i} k+\beta^{m+2} k /(1-\beta)=k \beta /(1-\beta)
$$

check bits formed by using $\mathcal{C}\left(B_{0}\right)$ to produce $\beta k$ check bits for the $k$ message bits, using $\mathcal{C}\left(B_{i}\right)$ to form $\beta^{i+1} k$ check bits for the $\beta^{i} k$ bits produced by $\mathcal{C}\left(B_{i-1}\right)$, and finally using $C$ to produce

\footnotetext{
${ }^{1}$ We will use the word bit in a rather loose form, mostly to denote coordinate positions.

${ }^{2}$ Herein lies one of the differences of our codes compared to Gallager's lowdensity parity-check codes: in the latter, the coordinate places of the codeword itself are identified with the left nodes, and the right nodes define constraints on these words. That is, Gallager allows only those words such that for any right node, the XOR of its adjacent left nodes is zero.
} 


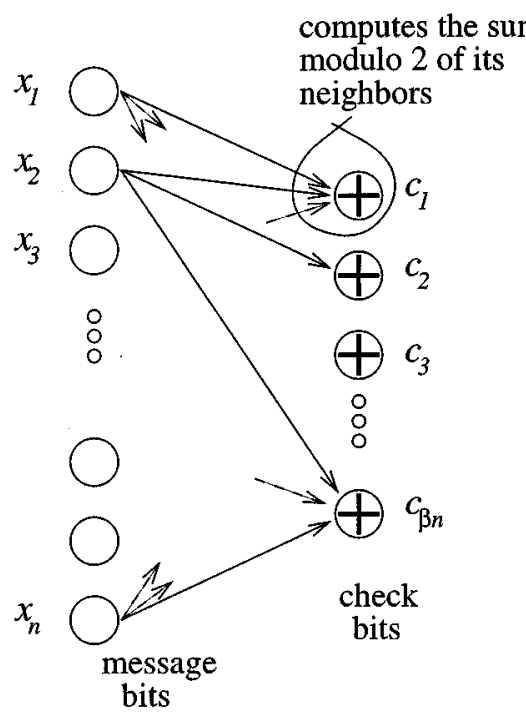

(a)

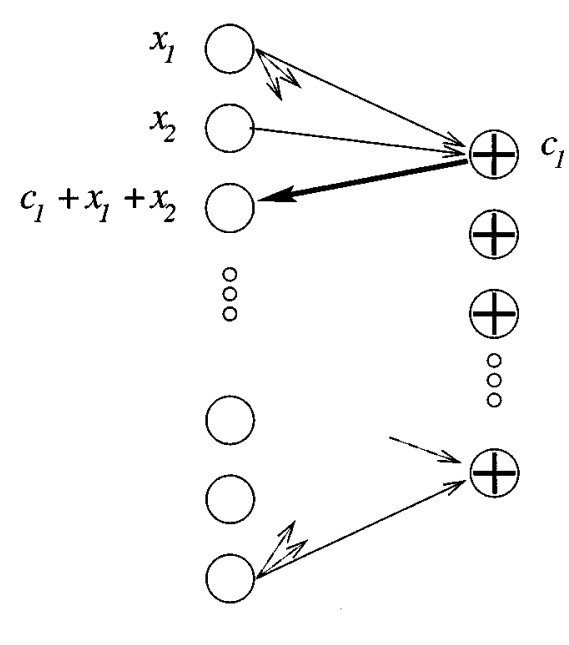

(b)

Fig. 1. (a) A graph defines a mapping from message bits to check bits. (b) Bits $x_{1}, x_{2}$, and $c_{1}$ are used to solve for $x_{3}$.

an additional $k \beta^{m+2} /(1-\beta)$ check bits for the $\beta^{m+1} k$ bits output by $\mathcal{C}\left(B_{m}\right)$. As $\mathcal{C}\left(B_{0}, B_{1}, \ldots, B_{m}, C\right)$ has $k$ message bits and $k \beta /(1-\beta)$ check bits, it is a code of rate $1-\beta$.

Remark 1: Assuming that the code $C$ can be encoded and decoded in quadratic time (an assumption which is certainly true for RS codes), the code $\mathcal{C}\left(B_{0}, \ldots, B_{m}, C\right)$ can be encoded and decoded in time proportional to the number of edges in all the $\mathcal{C}\left(B_{i}\right) .^{3}$

We begin by using the decoding algorithm for $C$ to decode erasures that occur within its corresponding message bits. If $C$ corrects all the erasures, then the algorithm now knows all the check bits produced by $\mathcal{C}\left(B_{m}\right)$, which it can then use to correct erasures in the inputs to $\mathcal{C}\left(B_{m}\right)$. As the inputs to each $\mathcal{C}\left(B_{i}\right)$ were the check bits of $\mathcal{C}\left(B_{i-1}\right)$, we can work our way back up the recursion until we use the check bits produced by $\mathcal{C}\left(B_{0}\right)$ to correct erasures in the original $k$ message bits. If we show that $C$ can correct a random $\beta(1-\epsilon)$ fraction of erasures with high probability, and that each $\mathcal{C}\left(B_{i}\right)$ can correct a random $\beta(1-\epsilon)$ fraction of erasures of its message bits with high probability, then we have shown that $\mathcal{C}\left(B_{0}, B_{1}, \ldots, B_{m}, C\right)$ is a rate $1-\beta$ code that can correct a random $\beta\left(1-\epsilon^{\prime}\right)$ fraction of erasures with high probability, for some $\epsilon^{\prime}$. Details can be found in the proof of Theorem 2.

For the remainder of this section and much of the next section, we only concern ourselves with finding graphs $B$ so that the decoding algorithm can correct $\beta(1-\epsilon)$ fraction of erasures in the message bits of $\mathcal{C}(B)$, given all of its check bits.

\section{B. The Graph Process and Degree Sequences}

We now relate the decoding process of $\mathcal{C}(B)$ to a process on a subgraph of $B$, so that hereafter we can use this simpler terminology when describing the process. This subgraph consists of

\footnotetext{
${ }^{3}$ If the alphabet size is too small for the corresponding RS code to exist, we can continue the cascade until the graph has roughly $\sqrt[4]{k}$ nodes and use a random linear code with conventional erasure decoding.
}

all nodes on the left that were erased but have not been decoded thus far, all the nodes on the right, and all the edges between these nodes. Recall that the decoding process requires finding a check bit on the right such that only one adjacent message bit is missing; this adjacent bit can then be recovered. In terms of the subgraph, this is equivalent to finding a node of degree one on the right, and removing it, its neighbor, and all edges adjacent to its neighbor from the subgraph. We refer to this entire sequence of events hereafter as one step of the decoding process. We repeat this step until there are no nodes of degree one available on the right. The entire process is successful if it does not halt until all nodes on the left are removed, or equivalently, until all edges are removed. It is simple to show that the result of this process is independent of the order in which nodes are removed; subsequently, in the analysis, we may freely assume that the nodes of degree one are chosen uniformly at random at each step.

The graphs that we use are chosen at random from a set of sparse bipartite graphs with a carefully chosen degree sequence. In contrast with many applications of random graphs in computer science, our graphs are not regular.

We refer to edges that are adjacent to a node of degree $i$ on the left (right) as edges of degree $i$ on the left (right). Each of our degree sequences is specified by a pair of vectors $\left(\lambda_{1}, \ldots, \lambda_{m}\right)$ and $\left(\rho_{1}, \ldots, \rho_{m}\right)$, where $\lambda_{i}$ is the initial fraction of edges on the left of degree $i$ and $\rho_{j}$ is the initial fraction of edges on the right of degree $j$. Note that we specify graphs in terms of fractions of edges, and not nodes, of each degree, as this form turns out to be more convenient. The sequences $\lambda$ and $\rho$ give rise to generating polynomials $\lambda(x)=\sum_{i} \lambda_{i} x^{i-1}$ and $\rho(x)=\sum_{i} \rho_{i} x^{i-1}$. The unusual choice of $x^{i-1}$ rather than $x^{i}$ has to do with the analysis of the decoding, as described below. Using these functions, one can succinctly describe many important parameters of the graph. For instance, it is easy to see that the average left degree $a_{\ell}$ of the graph is $\frac{1}{\sum_{i} \lambda_{i} / i}$ which is $\frac{1}{\int_{0}^{1} \lambda(x) d x}$. If $E$ is the number of edges in the graph, then the number of left nodes of degree $i$ is $E \lambda_{i} / i$, and hence the number of left nodes is $E \sum_{i} \lambda_{i} / i$. 


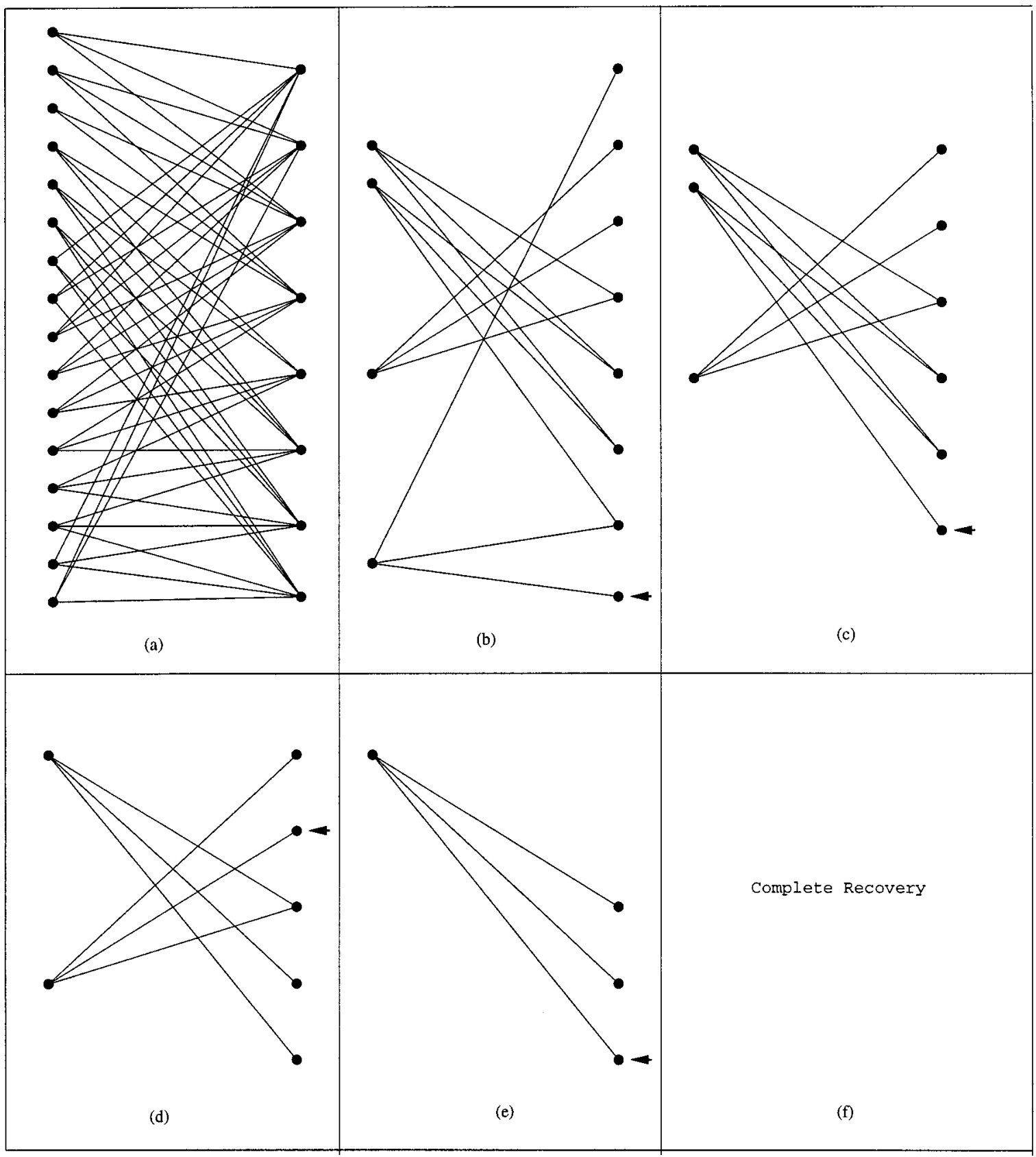

Fig. 2. All stages of the recovery. (a) Original graph. (b) Graph induced by the set of lost nodes on the left. (c)-(f) Recovery process.

Hence, the average degree is $E$ divided by this quantity. By a similar reasoning, the polynomial $\Lambda(x)=\int_{0}^{x} \lambda(t) d t / a_{\ell}$ has the property that its $i$ th coefficient is the fraction of left nodes of degree $i$. (Analogous assertions hold of course for $\rho(x)$.)

For a given pair $\lambda(x)$ and $\rho(x)$ of degree sequences, we will be interested in constructing a random bipartite graph with $k$ nodes on the left and $\beta k$ nodes on the right which has this degree distribution. We will implicitly assume that the numbers work out, i.e., that $\beta k, E \lambda_{i} / i$, and $E \rho_{i} / i$ are integers for all $i$, and we assume that $\beta \int_{0}^{1} \rho(x) d x=\int_{0}^{1} \lambda(x) d x$. In this case, it is easy to see that such graphs exist (say by induction). Later, in Section V-C, we will carry out a procedure to uniformly sample graphs (with multiedges) from the set of graphs with given degree sequences $\lambda$ and $\rho$.
Note that, as the decoding process evolves, in the corresponding subgraph $B^{\prime}$ of $B$ remaining after each step the matching remaining on $B^{\prime}$ still corresponds to a random permutation. Hence, conditioned on the degree sequence of the remaining subgraph after each step, the subgraph that remains is uniform over all subgraphs with this degree sequence. The evolution of the degree sequence is therefore a Markov process, a fact we make use of below.

In the next two sections, we develop techniques for the analysis of the process for general degree sequences.

\section{Large Deviation and Analysis of the Decoding}

We analyze the decoding algorithm (Algorithm 1) by viewing it as a discrete random process. We model the evolution of the 


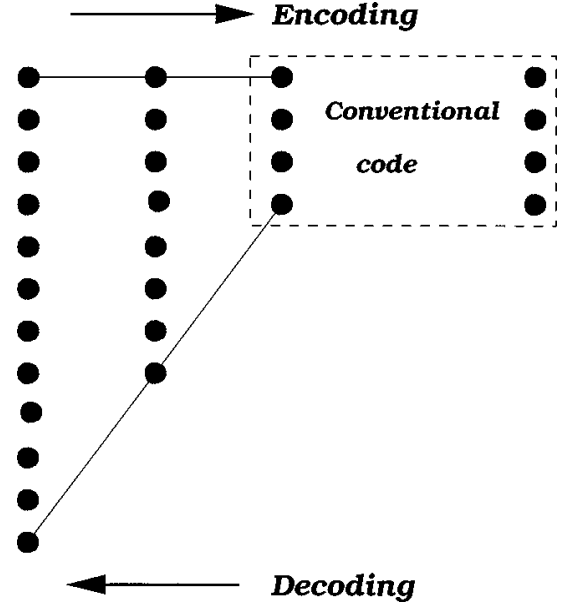

Fig. 3. The code levels and directions of encoding and decoding process.

main parameters of this system by a system of differential equations. These parameters include the number of edges of different right and left degrees, as well as the total number of edges and the average degrees of the bipartite graph on both sides. We need a result which makes sure that these parameters are sharply concentrated around the solutions of the system of equations, in the sense that the variation in the parameters are small compared with the total number of steps. For the sake of keeping the technical discussion at an acceptable level, we do not aim for the best possible results on the quality of the sharpness of the concentration.

In the first part of this section, we state a general large deviation result which we will prove in Appendix A. Similar results were obtained by Kurtz [8] who studied Markov jump processes, and have been used previously by many researchers, see [5], [7], [17], [18], [20], [26] and the references therein. We use a version due to Wormald [26] which has the advantage of being directly applicable to our situation.

Next we set up the appropriate system of differential equations, and solve them explicitly. This provides us with a concrete condition on the bipartite graph for successful decoding. However, we can only make limited use of the large deviation result, as this only guarantees continuation of the recovery process as long as the number of edges in the induced subgraphs is a constant fraction of the original number of edges. To prove that the process ends successfully, we need a combinatorial argument which proves that the random graph obtained at this stage of the decoding has reasonable expansion properties, with high probability. This expansion property suffices to show that once the number of edges remaining becomes sufficiently small, the decoding process is completed.

\section{A. Large Deviation}

For analyzing our erasure decoding algorithm we need to keep track of nodes of degree one on the right side of the bipartite graph as the algorithm proceeds. As the erasures occur randomly on the left side, it is not surprising that the analysis requires tools from probability theory. We may regard the number of edges of different degrees on the left and the right sides of the graph as random variables that evolve over time. It is relatively easy to compute the conditional expectation of these random variables. This is done in the next subsection. What we need is a tool that asserts that these random variables do not deviate too much from their expected value over the lifetime of the process. This is a typical example of a so-called large deviation result which we derive in this subsection. We assume that the reader is familiar with basic concepts such as (super- and sub-)martingales [19]. For this argument, we follow [26] rather closely.

The evolution of the number of edges of different degrees on the graphs considered is a typical example of a discrete-time random process. Let $\Omega$ denote a probability space and $S$ a measurable space. A discrete-time random process over $\Omega$ with state space $S$ is a sequence $Q=\left(Q_{0}, Q_{1}, \ldots\right)$ of random variables $Q_{i}: \Omega \rightarrow S$. To every $\omega \in \Omega$ corresponds a realization $\left(Q_{0}(\omega), Q_{1}(\omega), \ldots\right)$ of the process. The history of the process up to time $t$ is the sequence $H_{t}=\left(Q_{0}, Q_{1}, \ldots, Q_{t}\right)$. For a real-valued measurable function $y$ defined on $S^{+}:=\bigcup_{i \geq 1} S^{i}$, the random variable $y\left(H_{t}\right)$ is denoted by $Y_{t}$.

We say that a function $f: \boldsymbol{R}^{j} \rightarrow \boldsymbol{R}$ satisfies a Lipschitz condition on $D \subseteq R^{j}$ if there exists a constant $L>0$ such that

$$
|f(u)-f(v)| \leq L \cdot \sum_{i=1}^{j}\left|u_{i}-v_{i}\right|
$$

for all $u, v \in D$.

For a sequence of real-valued random variables $X_{m}$ taking only a countable number of values, we say that $X_{m}=O(f(m))$ with probability 1 , if

$$
\sup \left\{x \mid \operatorname{Pr}\left(X_{m}=x\right) \neq 0\right\}=O(f(m)) .
$$

The following theorem summarizes the large deviation result we need later. Its proof can be found in Appendix A.

Theorem 1: Let $\left(Q^{(m)}\right)_{m \geq 1}$ be a sequence of discrete time random processes

$$
Q^{(m)}=\left(Q_{0}^{(m)}, Q_{1}^{(m)}, \ldots\right)
$$

over a probability space $\Omega$ with state space $S_{m}$ and

$$
H_{t}^{(m)}:=\left(Q_{0}^{(m)}, \ldots, Q_{t}^{(m)}\right)
$$

be the $m$ th history up to time $t$. Let $d$ be a positive integer. For $1 \leq i \leq d$ and all positive integers $m$ let $y^{(i, m)}: S_{m}^{+} \rightarrow$ $\boldsymbol{R}$ be a measurable function such that $\left|y^{(i, m)}(h)\right|<C m$ for all $h \in S_{m}^{+}$and for some constant $C$ (independent of $i, m$, $h$ ). Furthermore, let $f_{1}, \ldots, f_{d}$ be functions from $\boldsymbol{R}^{d+1}$ to $\boldsymbol{R}$. Suppose

i) there exists a constant $C^{\prime}$ such that for all $m$, for all $t<$ $m$, and for all $i \leq d$

$$
\left|Y_{t+1}^{(i, m)}-Y_{t}^{(i, m)}\right|<C^{\prime}
$$

where $Y_{t}^{(i, m)}:=y^{(i, m)}\left(H_{t}^{(i, m)}\right)$;

ii) for all $i$ and uniformly over all $(m, t)$ with $t<m$ we have

$$
\begin{aligned}
\boldsymbol{E}\left(Y_{t+1}^{(i, m)}-Y_{t}^{(i, m)} \mid H_{t}\right) \\
=f_{i}\left(t / m, Y_{t}^{(1, m)} / m, \ldots, Y_{t}^{(d, m)} / m\right)
\end{aligned}
$$


iii) for each $i \leq d$, the function $f_{i}$ is continuous and satisfies a Lipschitz condition on $D$, where $D$ is some bounded connected open set containing the intersection of $\left\{\left(t, z_{1}, \ldots, z_{d}\right) \mid t \geq 0\right\}$ with some open neighborhood

$$
\begin{aligned}
&\left\{\left(0, z_{1}, \ldots, z_{d}\right) \mid \operatorname{Pr}\left(Y_{0}^{(i, m)}\right.\right. \\
&\left.\left.=z_{i} m \mid 1 \leq i \leq d\right) \neq 0 \text { for some } m\right\} .
\end{aligned}
$$

Then the following holds.

a) For $\left(0, \zeta_{1}, \ldots, \zeta_{d}\right) \in D$, the system of differential equations

$$
\frac{d z_{i}}{d \tau}=f_{i}\left(\tau, z_{1}, \ldots, z_{d}\right), \quad i=1, \ldots, d
$$

has a unique solution in $D$ for $z_{i}: R \rightarrow R$ passing through $z_{i}(0)=\zeta_{i}, 1 \leq i \leq d$

b) There is a constant $c$ such that

$$
\begin{aligned}
\operatorname{Pr}\left(Y_{t}^{(i)}>m z_{i}(t / m)+c m^{5 / 6}\right) & \\
& <d m^{2 / 3} \exp (-\sqrt[3]{m} / 2)
\end{aligned}
$$

for $0 \leq t \leq \sigma m$ and for each $i$, where $z_{i}(t)$ is the solution in a) with $\zeta_{i}=\boldsymbol{E}\left(Y_{0}^{(i)}\right) / m$, and $\sigma=\sigma(m)$ is the supremum of those $\tau$ to which the solution can be extended.

\section{B. The Differential Equations}

We begin with the initial random graph $B$, with $k$ left nodes and $\beta k$ right nodes. Suppose that the graph is given by the degree distribution pair $\lambda(x)$ and $\rho(x)$, as explained in Section II-B, and suppose that the total number of edges in the graph is $E$. As was explained above, the average node degree $a_{\ell}$ on the left initially satisfies $a_{\ell}^{-1}=\sum_{i} \lambda_{i} / i$, and similarly the average node degree $a_{r}$ on the right initially satisfies $a_{r}^{-1}=\sum_{i} \rho_{i} / i$.

In the application of Theorem 1, each time step corresponds to recovering one node on the left-hand side. Furthermore, the parameter $m$ corresponds to the total number $E$ of edges. Let $\delta$ be the fraction of erasures in the message. Initially, just prior to time 0 , each node on the left is removed with probability $1-\delta$ (because the corresponding message bit is successfully received), and thus the initial subgraph of $B$ contains $\delta k$ nodes on the left. If the process terminates successfully, it runs until time $\delta k=E \delta / a_{\ell}$. We denote by $\tau$ the scaled time $t / E$. Hence, $\tau$ runs from 0 to $\delta / a_{\ell}$.

Let $G$ be the graph obtained after a random deletion of ( $1-$ $\delta) k$ nodes on the left. We let $Q_{t}$ be the $t$ th edge removed in the graph $G$. We define $G_{t}$ to be the graph obtained after removing $Q_{1}, \ldots, Q_{t}$, all left nodes they are connected to, and all edges coming out of these nodes. If the process has already stopped at time $t-1$, we set $G_{t}=G_{t-1}$ for convenience.

We denote by $\mathcal{L}_{t}^{(i)}$ the number of edges of left degree $i$ at time $t$, and by $\mathcal{R}_{t}^{(i)}$ the number of edges of right degree $i$ at time $t$. Let $E$ be the total number of edges in the original graph. We let $\ell_{t}^{(i)}:=\mathcal{L}_{t}^{(i)} / E$ and $r_{t}^{(i)}:=\mathcal{R}_{t}^{(i)} / E$ represent the fraction of edges (in terms of $E$ ) of degree $i$ on the left and right, respectively, at time $t$. We denote by $e_{t}$ the fraction of the edges remaining at time $t$, that is, $e_{t}=\sum_{i} \ell_{t}^{(i)}=\sum_{i} r_{t}^{(i)}$.

First we note that $\left|\mathcal{L}_{t+1}^{(i)}-\mathcal{L}_{t}^{(i)}\right| \leq i$ for all $i$ and $t$, so Condition i) in Theorem 1 is satisfied. Recall that at each step, a

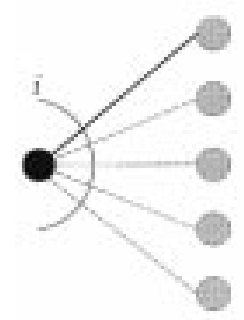

(a) Lef Side
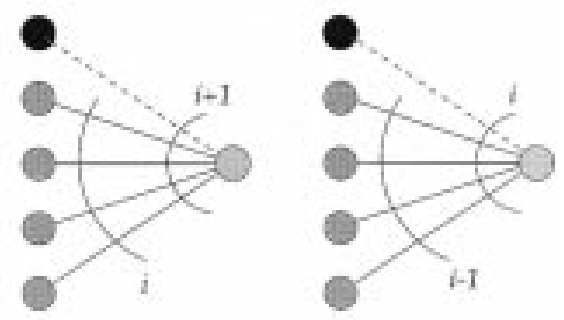

(b) Right sife
Fig. 4. Description of the differential equations.

random node of degree one on the right is chosen, and the corresponding node on the left and all of its adjacent edges are deleted. (If there is no such node, the process necessarily stops.) The probability that the edge adjacent to the node of degree one on the right has degree $i$ on the left is $\ell_{t}^{(i)} / e_{t}$, and in this case we lose $i$ edges of degree $i$, see Fig. 4(a). Hence, we have

$$
E\left(\mathcal{L}_{t+1}^{(i)}-\mathcal{L}_{t}^{(i)} \mid H_{t}\right)=-\frac{i \ell_{t}^{(i)}}{e_{t}}
$$

for $i=1, \ldots, d$, where $d$ is the maximum degree on the left-hand side.

Abusing the notation slightly, we set

$$
f_{i}\left(s, \ell_{1}, \ldots, \ell_{d}\right):=-\frac{i \ell_{i}}{e}
$$

where $e=\sum_{j} \ell_{j}$, and see that Condition ii) of Theorem 1 is satisfied for these functions. Furthermore, fixing $\eta>0$, we further see that these functions satisfy Condition iii) in the domain $D$ defined by the inequalities $0<s<\delta+\eta, 0<\ell_{i}<1+\eta$, for all $i=1, \ldots, d$, and $\eta<e<\delta+\eta$. Now Theorem 1 implies that with high probability we have

$$
\mathcal{L}_{t}^{(i)}=E \ell_{i}(t / E)+O\left(E^{5 / 6}\right)
$$

uniformly for all $E \eta \leq t \leq(\delta+\eta) E$, where $\ell_{i}(\tau)$ form the solution to

$$
\frac{d \ell_{i}(\tau)}{d \tau}=-\frac{i \ell_{i}(\tau)}{e(\tau)}
$$

for $i=1, \ldots, d$.

These differential equations are solved by defining $x$ so that $d x / d \tau=-x / e(\tau)$. The value of $x$ in terms of $\tau$ is then

$$
x:=\exp \left(-\int_{0}^{\tau} d s / e(s)\right) .
$$

By substituting $d x / x$ for $d \tau / e(\tau),(1)$ becomes $d \ell_{i}(x) / d x=$ $i \ell_{i}(x) / x$, and integrating yields $\ell_{i}(x)=c_{i} x^{i}$. Note that $x=1$ for $t=0$, and $\ell_{i}(\tau=0)=\delta \lambda_{i}$. Hence, $c_{i}=\delta \lambda_{i}$ and

$$
\ell_{i}(x)=\delta \lambda_{i} x^{i} .
$$

Since $\ell_{i}(x)$ goes to zero as $\tau$ goes to $\delta / a_{\ell}, x$ runs over the interval $(0,1]$.

To discuss the evolution of the right-hand side, first note that $\left|\mathcal{R}_{t+1}^{(j)}-\mathcal{R}_{t}^{(j)}\right|<d$, where $d$ is the maximum degree on the left. This is because a left node is connected to at most $d$ right nodes and one of the right neighbors has been used to recover the left node. Hence, Condition i) of the theorem is satisfied. Note that when we remove a node of degree $i$ on the left, we remove the one edge of degree one from the right, along with the $i-1$ 
other edges adjacent to this node. Hence the expected number of other edges deleted is $a_{t}-1$, where $a_{t}=\sum i \ell_{t}^{(i)} / e_{t}$. The right endpoints of these $i-1$ other edges on the right-hand side are randomly distributed. If one of these edges is of degree $j$ on the right, we lose $j$ edges of degree $j$, and gain $j-1$ edges of degree $j-1$, see Fig. 4(b). The probability that an edge has degree $j$ on the right is just $r_{t}^{(j)} / e_{t}$. Then, for $i>1$, we have

$$
\boldsymbol{E}\left(\mathcal{R}_{t+1}^{(i)}-\mathcal{R}_{t}^{(i)} \mid H_{t}\right)=\left(r_{t}^{(i+1)}-r_{t}^{(i)}\right) \frac{i\left(a_{t}-1\right)}{e_{t}} .
$$

(We assume that $r_{t}(i)$ is defined for all positive $i$, and is 0 for sufficiently large $i$.) The case $i=1$ plays a special role, as we must take into account that at each step an edge of degree one on the right is removed. This gives

$$
\boldsymbol{E}\left(\mathcal{R}_{t+1}^{(1)}-\mathcal{R}_{t}^{(1)} \mid H_{t}\right)=\left(r_{t}^{(2)}-r_{t}^{(1)}\right) \frac{\left(a_{t}-1\right)}{e_{t}}-1 .
$$

Let $\mu$ be the maximum degree of a node on the right. Abusing the notation slightly, we set

$$
\begin{aligned}
g_{\mu}\left(\tau, r_{1}, \ldots, r_{\mu}\right) & :=-r_{\mu} \frac{\mu(a-1)}{e} \\
g_{i}\left(\tau, r_{1}, \ldots, r_{\mu}\right) & :=\left(r_{i+1}-r_{i}\right) \frac{i(a-1)}{e}, \quad \text { for } 1<i<\mu \\
g_{1}\left(\tau, r_{1}, \ldots, r_{\mu}\right) & :=\left(r_{2}-r_{1}\right) \frac{(a-1)}{e}-1
\end{aligned}
$$

where $e=\sum_{j} r_{j}$ and $a:=\sum_{j} j \ell_{j} / e$. Fixing any $\eta>0$, we see as in the case of the left-hand side evolution, that these functions satisfy a Lipschitz condition, as long as $\tau=t / E>\eta$. Application of Theorem 1 thus yields that almost surely, we have

$$
\mathcal{R}_{t}^{(i)}=E r_{i}(t / E)+O\left(E^{5 / 6}\right)
$$

uniformly for all $\eta E \leq t \leq(\delta+\eta) E$, where $r_{i}(\tau)$ form the solution to

$$
\frac{d r_{i}(\tau)}{d t}=\left(r_{i+1}(\tau)-r_{i}(\tau)\right) \frac{i(a(\tau)-1)}{e(\tau)}, \quad \text { for } i>1
$$

and

$$
\frac{d r_{1}(\tau)}{d \tau}=\left(r_{2}(\tau)-r_{1}(\tau)\right) \frac{(a(\tau)-1)}{e(\tau)}-1
$$

Our key interest is in the progression of $r_{1}(\tau)$ as a function of $\tau$. As long as $r_{1}(\tau)>0$, so that we have a node of degree one on the right, the process continues; when $r_{1}(\tau)=0$ the process stops. Hence we would like $r_{1}(\tau)>0$ until all nodes on the left are deleted and the process terminates successfully.

We proceed with the determination of $r_{j}(1)$, the expected fraction of edges of right degree one at time 0 : because each node on the left is deleted randomly just prior to time 0 with probability $1-\delta$, and the graph is a random graph over those with the given degree sequence, to the nodes on the right it is as though each edge is deleted with probability $1-\delta$. Hence, an edge whose right incident node had degree $j$ before the deletion stage remains in the graph and has degree $i$ afterwards with probability $\left(\begin{array}{c}j-1 \\ i-1\end{array}\right) \delta^{i}(1-\delta)^{j-i}$. Thus

$$
r_{j}(1)=\sum_{m \geq j} \rho_{m}\left(\begin{array}{c}
m-1 \\
j-1
\end{array}\right) \delta^{j}(1-\delta)^{m-j} .
$$

In Appendix B we will solve the set of differential equations given by (3) and (4) with the initial condition (5). Here is the result.

Proposition 1: For the solution to the system of differential equations given by (3) and (4) with the initial condition (5) we have

$$
r_{1}(x)=\delta \lambda(x)[x-1+\rho(1-\delta \lambda(x))]
$$

where $x$ is defined via $d x / d \tau=-x / e(\tau)$.

This immediately gives rise to the following result.

Proposition 2: Let $B$ be a bipartite graph with $k$ message bits that is chosen at random with edge degrees specified by $\lambda(x)$ and $\rho(x)$. Let $\delta$ be fixed so that

$$
\rho(1-\delta \lambda(x))>1-x, \quad \text { for } x \in(0,1] .
$$

For all $\eta>0$ there is some $k_{0}$ such that for all $k \geq k_{0}$, if the message bits of $\mathcal{C}(B)$ are erased independently with probability $\delta$, then with probability at least $1-k^{2 / 3} \exp (-\sqrt[3]{k} / 2)$ the recovery algorithm terminates with at most $\eta k$ message bits erased.

Proof: Let $E$ be the number of edges in the graph. Then $E=k a_{\ell}$, where $a_{\ell}$ is the average degree of the nodes in the left side of the graph, which is a constant for fixed $\lambda$ and $\rho$. (Note that $a_{\ell}=\sum \lambda_{i} / i$.) Let $\mu:=\eta / a_{\ell}$. By (6) and the preceding discussions, with probability at least $1-k^{2 / 3} \exp (-\sqrt[3]{k} / 2)$ the number of nodes of degree one on the right is

$$
\delta \lambda(x)[x-1+\rho(1-\delta \lambda(x))]+O\left(k^{5 / 6}\right)
$$

for $x \in\left(\eta^{\prime}, 1\right]$, where $\eta^{\prime}=\exp \left(-\int_{0}^{\mu} d s / e(s)\right)$. By our assumption, this number is positive (for large enough $k$ ), which proves the assertion.

The foregoing proposition does not prove that the decoding process terminates successfully recovering all the missing nodes on the left-hand side. To do this, we need a combinatorial argument which says that random graphs are good expanders. This means that any small enough subset of left nodes has many right neighbors. The exact statement is given in the proof of the following result.

Lemma 1: Let $B$ be a bipartite graph with $k$ left nodes chosen at random with edge degrees specified by $\lambda(x)$ and $\rho(x)$, such that $\lambda(x)$ has $\lambda_{1}=\lambda_{2}=0$. Then there is some $\eta>0$, such that, with probability $1-O\left(k^{-3 / 2}\right)$, the recovery process restricted to the subgraph induced by any $\eta$-fraction of the left nodes terminates successfully.

Proof: Let $S$ be any set of nodes on the left of size at most $\eta k$, where $\eta$ will be chosen later. Let $a$ be the average degree of these nodes. If the number of nodes on the right that are neighbors of $S$ is greater than $a|S| / 2$, then one of these nodes has only one neighbor in $|S|$, and so the process can continue. Thus, we only need to show that the initial graph is a good expander on small sets.

Let $\mathcal{E}_{s}$ denote the event that a subset of size $s$ of the nodes on the left has at most $a s / 2$ neighbors. We first bound $\operatorname{Pr}\left(\mathcal{E}_{s}\right)$, and then sum $\operatorname{Pr}\left(\mathcal{E}_{s}\right)$ over all values of $s$ no larger than $\eta k$. Fix any subset $S$ of the left nodes of size $s$, and any subset $T$ of the right nodes of size $a s / 2$. There are $\left(\begin{array}{l}k \\ s\end{array}\right)$ ways of choosing $S$, and 
$\left(\begin{array}{c}\beta k \\ a s / 2\end{array}\right)$ ways of choosing $T$. The probability that $T$ contains all the $a s$ neighbors of the vertices in $S$ is $(a s / 2 \beta k)^{a s}$. Hence, we have

$$
\operatorname{Pr}\left(\mathcal{E}_{s}\right) \leq\left(\begin{array}{c}
k \\
s
\end{array}\right)\left(\begin{array}{c}
\beta k \\
a s / 2
\end{array}\right)\left(\frac{a s}{2 \beta k}\right)^{a s} .
$$

Note that $\left(\begin{array}{l}n \\ k\end{array}\right) \leq(n e / k)^{k}$, hence we have

$$
\operatorname{Pr}\left(\mathcal{E}_{s}\right) \leq\left(\frac{s}{k}\right)^{(a / 2-1) s} c^{s} \leq\left(\frac{s c^{2}}{k}\right)^{s / 2}
$$

where $c$ is a constant (depending on $\beta$ and $a$ ). Since the graph does not have nodes of degree one or two, we have that $\operatorname{Pr}\left(\mathcal{E}_{1}\right)=\operatorname{Pr}\left(\mathcal{E}_{2}\right)=0$. Choosing $\eta \leq 1 /\left(2 c^{2}\right)$ yields

$$
\begin{aligned}
\sum_{s=1}^{\eta k} \operatorname{Pr}\left(\mathcal{E}_{s}\right) & \leq \sum_{s=3}^{\eta k}\left(\frac{s c^{2}}{k}\right)^{s / 2} \leq \frac{3 c^{2}}{k \sqrt{k}}+\sum_{s=4}^{\eta k} \frac{1}{2^{s}} \\
& =O\left(\frac{1}{k \sqrt{k}}\right)
\end{aligned}
$$

which shows that, with high probability, the original graph is an expander on small subsets.

The above proof shows that the main contribution for the error probability comes from nodes of degree three on the left. For the same reason, it is easy to see that nodes of degree two will lead to a constant error probability. We leave the details of this argument to the reader.

Altogether we obtain the main theorem of this section.

Theorem 2: Let $k$ be an integer, and suppose that

$$
\mathcal{C}=\mathcal{C}\left(B_{1}, \ldots, B_{m}, C\right)
$$

is a cascade of bipartite graphs as explained in Section II, where $B_{1}$ has $k$ left nodes. Suppose that each $B_{i}$ is chosen at random with edge degrees specified by $\lambda(x)$ and $\rho(x)$, such that $\lambda(x)$ has $\lambda_{1}=\lambda_{2}=0$, and suppose that $\delta$ is such that

$$
\rho(1-\delta \lambda(x))>1-x
$$

for all $0<x \leq 1$. Then, if at most a $\delta$-fraction of the coordinates of an encoded word in $\mathcal{C}$ are erased independently at random, our erasure decoding algorithm terminates successfully with probability $1-O\left(k^{-3 / 4}\right)$, and does so in $O(k)$ steps.

Proof: At each level of the cascade, the number of edges equals the average degree of the nodes on the left times the number of the nodes. The average degree is always $1 / \int_{0}^{1} \lambda(t) d t$, which is a constant. Hence, the total number of edges in the cascade (up to the last layer) is $O(k)$, which shows that the recovery process needs $O(k)$ steps (see Remark 1 ).

Next we bound the probability that there is some $j$ such that the fraction of left nodes lost on the left side of the graph $B_{j}$ is larger than $\delta^{\prime}:=\delta+1 / \sqrt[8]{k}$. We use a version of the Chernoff bounds given in [19, Problem 4.7(c), p. 98]. According to that, for any $j$, the probability that there are more erasures than $\delta\left(k / 2^{j}\right)+\left(k / 2^{j}\right)^{3 / 4}$ is upper-bounded by $\exp \left(-2 \sqrt{k / 2^{j}}\right)$, which is smaller than $\exp (-2 \sqrt[4]{k})$. The required probability is certainly at most equal to the sum of these probabilities (union bound), which is $\log (k) \exp (-\sqrt[4]{k}) / 2$. (Note that there are $\log (k) / 2 \operatorname{such} j$ 's.)
For large enough $k$, Condition (7) is satisfied for $\delta^{\prime}$ instead of $\delta$ (by continuity). Hence, invoking Proposition 2 , for any $\eta>0$ and any of the graphs $B_{j}$ in the cascade our decoding algorithm stops with less than $\eta k / 2^{j}$ nodes uncorrected, with probability $1-O\left(\exp \left(-k^{\gamma}\right)\right)$ for some positive $\gamma$. Now Lemma 1 applies and shows that, for small enough $\eta$, the recovery process ends successfully with probability $1-O\left(\left(2^{j} / k\right)^{3 / 2}\right)$. The probability that our algorithm fails on at least one of the graphs is thus at most $\sum_{j}\left(2^{j} / k\right)^{3 / 2}$, where $j$ runs from 0 to $\log (k) / 2$. This is equal to $O\left(k^{-3 / 4}\right)$, which shows the assertion.

For designing graphs that lead to good codes, it is thus necessary to fulfill condition (7). It is sometimes desirable to use the "dual condition"

$$
\delta \lambda(1-\rho(y))<1-y
$$

for $y \in[0,1)$, which is obtained from (7) by substituting $y:=$ $\rho^{-1}(1-x)$. Note that $\rho$ has an inverse on $(0,1]$, as it is monotonically increasing.

In the following section we use this theorem to analyze decoding properties of codes obtained from regular graphs.

\section{CAPACITY-ACHIEVING CODES}

In this section we will construct for any erasure probability $p$ families of codes with linear time erasure decoding algorithms that can correct any $p$-fraction of erasures and whose rates come arbitrarily close to the capacity $1-p$ of the erasure channel. In other words, we construct codes that are close to optimal in terms of their erasure recovery rate, and have linear time encoding and decoding algorithms. We do this by finding an infinite family of solutions to the differential equations of Section III in which $\delta$ is close to $1-R$, where $R$ is the rate.

Let $B$ be a bipartite graph with $k$ left nodes and $\beta k$ right nodes. We describe our choice for the left and right degree sequences of $B$ that satisfy condition (7). Let $D$ be a positive integer that is used to trade off the average degree with how well the decoding process works, i.e., how close we can make $\delta$ to $\beta=1-R$ and still have the process finish successfully most of the time.

The left degree sequence is described by the following truncated heavy tail distribution. Let $H(D)=\sum_{i=1}^{D} 1 / i$ be the harmonic sum truncated at $D$, and thus $H(D) \sim \ln (D)$. Then, for all $i=2, \ldots, D+1$, the fraction of edges of degree $i$ on the left is given by

$$
\lambda_{i}:=1 /(H(D)(i-1))
$$

The average left degree $a_{\ell}$ equals $H(D)(D+1) / D$. Recall that we require the average right degree $a_{r}$ to satisfy $a_{r}=a_{\ell} / \beta$. The right degree sequence is defined by the Poisson distribution with mean $a_{r}$ : for all $i \geq 1$ the fraction of edges of degree $i$ on the right equals

$$
\rho_{i}=\frac{e^{-\alpha} \alpha^{i-1}}{(i-1) !}
$$

where $\alpha$ is chosen to guarantee that the average degree on the right is $a_{r}$. In other words, $\alpha$ satisfies $\alpha e^{\alpha} /\left(e^{\alpha}-1\right)=a_{r}$.

Note that we allow $\rho_{i}>0$ for all $i \geq 1$, and hence $\rho(x)$ is not truly a polynomial, but a power series. However, truncating 
the power series $\rho(x)$ at a sufficiently high term gives a finite distribution of the edge degrees for which the next lemma is still valid.

We show that when $\delta=\beta(1-1 / D)$, then condition (7) is satisfied, i.e., $\rho(1-\delta \lambda(x))>1-x$ on $(0,1]$, where

$$
\lambda(x)=\sum_{i} \lambda_{i} x^{i-1}
$$

and

$$
\rho(x)=\sum_{i} \rho_{i} x^{i-1}
$$

Note that $\lambda(x)$ is the expansion of $-\ln (1-x)$ truncated at the $D$ th term, and scaled so that $\lambda(1)=1$. Further, $\rho(x)=e^{\alpha(x-1)}$.

Lemma 2: With the above choices for $\rho(x)$ and $\lambda(x)$ we have $\rho(1-\delta \lambda(x))>1-x$ on $(0,1]$ if $\delta \leq \beta /(1+1 / D)$.

Proof: Since $\rho(x)$ increases monotonically in $x$, we have

$$
\rho(1-\delta \lambda(x))>\rho(1+\delta \ln (1-x) / H(D))=(1-x)^{\alpha \delta / H(D)} .
$$

As $a_{\ell}=H(D)(1+1 / D)$ and $a_{r}=a_{\ell} / \beta$, we obtain

$$
\alpha \delta / H(D)=\left(1-e^{-\alpha}\right)(1+1 / D) \delta / \beta<\delta(1+1 / D) / \beta \leq 1
$$

which shows that the right-hand side of the above inequality is larger than $1-x$ on $(0,1]$.

A problem is that Lemma 1 does not apply to this system because there are nodes of degree two on the left. Indeed, simulations demonstrate that for these choices of $\lambda(x)$ and $\rho(x)$ a small number of nodes often do remain. To overcome this problem, we make a small change in the structure of the graph $B$. Let $\gamma:=\beta / D^{2}$. We split the $\beta k$ right nodes of $B$ into two distinct sets, the first set consisting of $(\beta-\gamma) k$ nodes and the second set consisting of $\gamma k$ nodes. The graph $B$ is then formed by taking the union of two graphs, $B_{1}$ and $B_{2} . B_{1}$ is formed as described up to this point between the $k$ left nodes and the first set of $(\beta-\gamma) k$ right nodes. $B_{2}$ is formed between the $k$ left nodes and the second set of $\gamma k$ right nodes, where each of the $k$ left nodes has degree three and the $3 k$ edges are connected randomly to the $\gamma k$ right nodes.

Lemma 3: Let $B$ be the bipartite graph described above. Then, with probability $1-O\left(k^{-3 / 2}\right)$, the decoding process terminates successfully when started on a subgraph of $B$ induced by $\delta k$ of the left nodes and all $\beta k$ of the right nodes, where $\delta=\beta(1-1 / D)$.

Proof: In the analysis of the process, we may think of $B_{2}$ as being held in reserve to handle nodes not already dealt with using $B_{1}$. First, using the same method as in Lemma 1 we can prove that there is some $\eta$ such that a set $S$ of $s \leq \eta k$ left nodes in the graph $B_{2}$ expands to a set of at least $3 s / 2$ nodes on the right, with probability $1-O\left(1 / k^{3 / 2}\right)$. (Note that all nodes on the left have degree three in this graph.) Combining Proposition 2 and Lemma 2, we see that the recovery process started on $B_{1}$ terminates with less than $\eta k$ nodes on the left unrecovered, with probability $1-O\left(\exp \left(-k^{a}\right)\right)$ for some positive $a$ : note that the ratio of the number of left nodes to the number of right nodes in the graph $B_{2}$ equals $\beta\left(1-1 / D^{2}\right)$, hence the condition in Lemma 2 translates to

$$
\delta \leq \beta\left(1-1 / D^{2}\right) /(1+1 / D)=\beta(1-1 / D)
$$

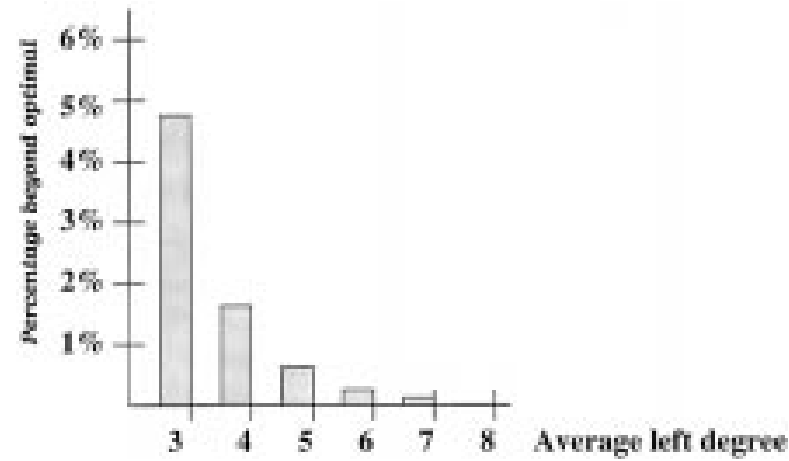

Fig. 5. Erasure decoding of irregular graphs: left degree versus the overhead beyond optimal value needed to recover $($ rate $=1 / 2)$.

which is obviously true. By the aforementioned expansion property of the subgraph of $B_{2}$ induced by the set of unrecovered left nodes, we see that the process terminates successfully.

Note that the degree of each left node in this modified construction of $B$ is at most three bigger than the average degree of each left node in the construction of $B$ described at the beginning of this section. We can use this observation and the lemma above to immediately prove the following.

Theorem 3: For any $R$ with $0<R<1$, any $\epsilon$ with $0<\epsilon<$ 1 , and sufficiently large block length $n$, there is a linear code and a decoding algorithm that, with probability $1-O\left(n^{-3 / 4}\right)$, is able to correct a random $(1-R)(1-\epsilon)$-fraction of erasures in time proportional to $n \ln (1 / \epsilon)$.

Proof: Set $D=\lceil 1 / \epsilon\rceil$ to get a one level code with the properties described in Lemma 3. Then cascade versions of these codes as described in Section II to get the entire code. As was pointed out above, the average degree $a_{\ell}$ of the left nodes in each of the layers is upper-bounded by $3+\sum_{i=1}^{D} 1 / i<4+\ln (1 / \epsilon)$, which is proportional to $\ln (1 / \epsilon)$. Hence, the total number of edges in the bipartite layers of the graph is proportional to $n \ln (1 / \epsilon)$, which proves the assertion on the decoding time.

Using Lemma 3 and the same analysis as in the proof of Theorem 2, we can show that the code constructed above can recover, with probability $1-O\left(n^{-3 / 4}\right)$, all the message bits, if a random $\delta$-fraction of the codeword is missing, where $\delta=$ $\beta(1-1 / D)$. Noting that $\beta=1-R$, we obtain the result.

Fig. 5 shows the running time of the encoding/recovery algorithms (as multiples of the block length) versus the overhead needed to recover. For instance, for sufficiently large $n$, one can construct in this way $[n, k]_{q}$-codes that have encoding/recovery algorithms running in time $\sim 7 n$, which can recover a codeword from a random set of $1.002 k$ of its coordinates.

\section{Practical Considerations}

The discussions in the preceding sections have been of a more theoretical, rather than a practical nature. However, as the graph codes designed via the above mentioned theorems can be used in practical situations, it is important to describe possible implementations. We start with modifying our construction by allowing erasures to also occur on the right-hand side. An analysis 
of this type provides us with some insight in how to design cascaded versions of our codes with much fewer levels, and faster decoding algorithms for the end level. Next, we show how to use a linear programming approach to design bipartite graphs which give rise to very good codes. Finally, we briefly discuss some of our implementations. A preliminary report on the results of this section appeared in [9].

\section{A. Fraction of Left Nodes Unrecovered}

So far we have assumed in our analysis that in each layer of the cascade all the check bits are received when trying to recover the message bits. The reason we made this assumption is that in the original construction the cascading sequence of bipartite graphs is completed by adding a standard erasure-correcting code at the last level.

There are some practical problems with this. One annoyance is that it is inconvenient to combine two different types of codes. A more serious problem is that standard erasure-correcting codes take quadratic time to encode and decode (if the alphabet size is large enough; otherwise, cubic running time will do). Suppose the message is mapped to a codeword twice its length. In order to have the combined code run in linear time, this implies that the last graph in the cascading sequence has $\sqrt{k}$ left nodes, where $k$ is the number of nodes associated with the original message, i.e., there are $O(\log (k))$ graphs in the sequence. In the analysis, we assume that an equal fraction of the nodes in each level of the graph are received. However, there is variance in this fraction at each level, with the worst expected fractional variance at the last level of $1 / \sqrt[4]{k}$. Thus, if a message of length 65536 is stretched to a codeword of length 131072 , then just because of the variance of $1 / \sqrt[4]{k}=0.063$, we expect to have to receive 1.063 times the message length of the codeword in order to recover the message.

A solution to this problem is to use many fewer levels of graphs in the cascade, and to avoid using a standard erasure-correcting code in the last level. That is, for the last layer, we continue to use a randomly chosen graph. We have tried this idea, with the last graph chosen from an appropriate distribution, and it works quite well. For example, using only three levels of graphs we can reliably recover a message of length 65536 from a random portion of length 67700 (i.e., 1.033 times the optimal of 65536 ) of a block-length of 131072 .

To design the graph for this solution, we need to analyze the decoding process when a random portion of both the message bits and the check bits are missing. The following result gives the expected fraction of right nodes of degree one with respect to the number of edges in the graph, and estimates the fraction of left nodes unrecovered at each step of the algorithm.

Lemma 4: Suppose each node on the left is missing with probability $\delta$ and each node on the right is missing with probability $\delta^{\prime}$. The fraction $r_{1}(x)$ of edges of right degree one at $x$ with respect to the number of edges in the original graph $B$ equals

$$
\begin{aligned}
& r_{1}(x)=\delta\left(1-\delta^{\prime}\right) \lambda\left(\delta^{\prime}+\right.\left.\left(1-\delta^{\prime}\right) x\right) \\
& \cdot\left[x-1+\rho\left(1-\delta \lambda\left(\delta^{\prime}+\left(1-\delta^{\prime}\right) x\right)\right)\right] .
\end{aligned}
$$

Furthermore, up to lower order terms, the fraction of left nodes unrecovered at time $x$ equals

$$
\delta a_{\ell} \cdot \int_{0}^{\left(\delta^{\prime}+\left(1-\delta^{\prime}\right) x\right)} \lambda(y) d y
$$

We will prove this lemma later in Appendix C. We immediately obtain the condition

$$
\rho\left(1-\delta \lambda\left(\delta^{\prime}+\left(1-\delta^{\prime}\right) x\right)\right)>1-x, \quad x \in(0,1]
$$

for successful decoding.

It is not possible to satisfy the above inequality for all $x \in$ $(0,1]$ if $\delta^{\prime}>0$, for any value of $\delta$ : for $x=0$ the left-hand side equals $\rho\left(1-\delta \lambda\left(\delta^{\prime}\right)\right)$ which is strictly less than 1 . There is an intuitive reason for this: the subgraph $\tilde{B}$ on which the process starts has edges of degree one on the left; these edges can only correct the left nodes they are connected to, and cannot help any other node on the left.

However, it turns out to be an interesting question to see what fraction of the left nodes can be recovered when a fraction $\delta^{\prime}$ of the right nodes is missing. The answer to this question can be used to design cascading codes where the decoding process moves from right to left bootstrapping up to recover a higher and higher fraction of nodes at each successive decoded layer of the graph until in practice it is able to recover all of the first (message) layer (see Fig. 6).

Given the $(\lambda)$ and $(\rho)$ vectors, condition (9) can be used to compute the smallest value of $x$ for which the condition is still valid. The second part of Lemma 4 then gives the fraction of unrecovered nodes on the left at this value of $x$.

\section{B. Computing Degree Sequences Using Linear Programming}

In this subsection, we describe a heuristic approach that has proven effective in practice to find a good right degree sequence given a specific left degree sequence. The method uses linear programming and the differential equation analysis of Section III. Recall that a necessary condition for the process to complete is that $\rho(1-\delta \lambda(x))>1-x$ on $(0,1]$. We first describe a heuristic for determining for a given $\lambda(x)$ representing the left degree sequence and a value for $\delta$ whether there is an appropriate $\rho(x)$ representing the right degree sequence satisfying this condition. We begin by choosing a set $M$ of positive integers which we want to contain the degrees on the right-hand side. To find appropriate $\rho_{m}, m \in M$, we use the condition given by Theorem 2 to generate linear constraints that the $\rho_{i}$ must satisfy by considering different values of $x$. For example, by examining the condition at $x=0.5$, we obtain the constraint $\rho(1-\delta \lambda(0.5))>0.5$, which is linear in the coefficients of $\rho(x)$.

We generate constraints by choosing for $x$ multiples of $1 / N$ for some integer $N$. We also include the constraints $\rho_{m} \geq 0$ for all $m \in M$. We then use linear programming to determine if suitable $\rho_{m}$ exist that satisfy our derived constraints. Note that we have a choice for the function we wish to optimize; one choice that works well is to minimize the sum of $\rho(1-\delta \lambda(x))+$ $x-1$ on the values of $x$ chosen to generate the constraints. The best value for $\delta$ for given $N$ is found by binary search. 

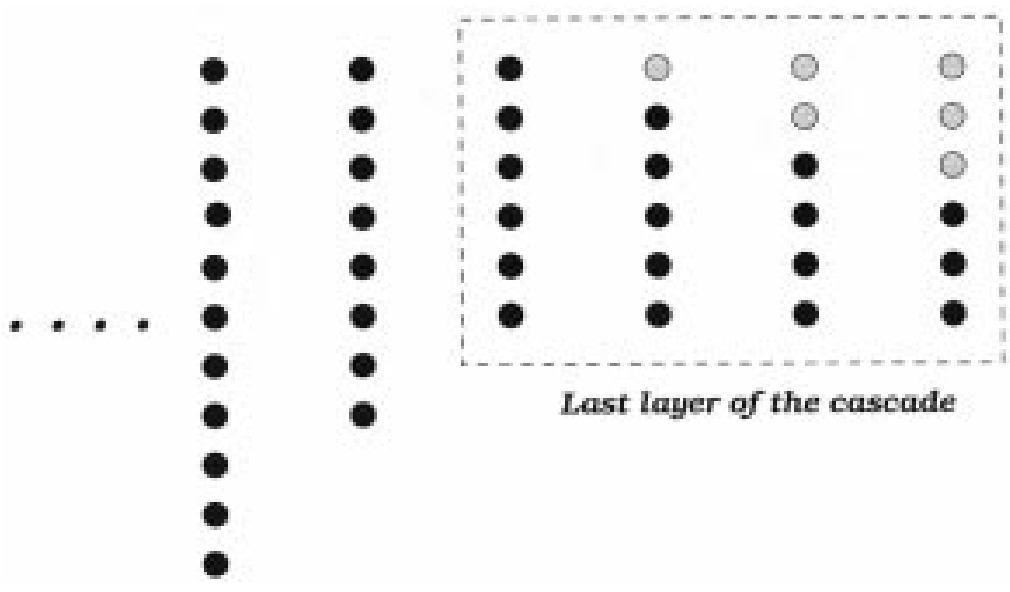

Last layer of the cascade

\section{Received node}

Lost node

Fig. 6. Bootstrapping strategy.

Given the solution from the linear programming problem, we can check whether the $\rho_{i}$ computed satisfy the condition $\rho(1-\delta \lambda(x))>1-x$ on $(0,1]$.

Due to our discretization, there are usually some conflict subintervals in which the solution does not satisfy this inequality. Choosing large values for the tradeoff parameter $N$ results in smaller conflict intervals, although it requires more time to solve the linear program. For this reason we use small values of $N$ during the binary search phase. Once a value for $\delta$ is found, we use larger values of $N$ for that specific $\delta$ to obtain small conflict intervals. In the last step, we get rid of the conflict intervals by appropriately decreasing the value of $\delta$. This always works since $\rho(1-\delta \lambda(x))$ is a decreasing function of $\delta$.

We ran the linear programming approach on left degree sequences of the form $3,5,9, \ldots, 2^{i}+1$ for codes with rates $1 / 2,2 / 3,3 / 4,4 / 5,9 / 10$ and average left degrees 5.70 , $6.82,8.01$. These results are gathered in Table I which shows how much of the codeword is sufficient to recover the entire message as a fraction of the message length as the message length goes to infinity. Since these graphs do not have nodes of degree two on the left, Theorem 2 implies that with high probability the corresponding codes recover the entire message from the portion of the codeword indicated in the table, provided the message length is large enough. However, as the maximum degrees in the examples we have found are rather large (about 30000 ), these codes are rather impractical.

One major disadvantage of the approach given above is that we need to fix the left-hand side of the graph. To overcome this difficulty, we use the dual condition (8). We can now use this condition and the linear programming approach to solve for the best $\lambda$ given $\rho$, then use the original condition to solve for the best $\rho$ given this $\lambda$, and so on. We have tried this strategy and it gives good results, although at this point we have not proved anything about its convergence to a (possibly optimal) pair of probability distributions.

For example, we found that the following pair of degree sequence functions yield $[2 k, k]_{q}$-codes which are able to recover from a random set of $1.01 k$ coordinates, with high probability;
TABLE I

Close to Optimal Codes for Different Rates and Average LEFT DEGREES

\begin{tabular}{|l||c|c|c|c|c|}
\hline \multicolumn{1}{|c||}{$\begin{array}{l}\text { Average } \\
\text { Degree }\end{array}$} & \multicolumn{5}{c|}{ Rate } \\
\cline { 2 - 6 } & $1 / 2$ & $2 / 3$ & $3 / 4$ & $4 / 5$ & $9 / 10$ \\
\hline 5.70 & 1.036 & 1.023 & 1.016 & 1.013 & 1.006 \\
\hline 6.82 & 1.024 & 1.013 & 1.010 & 1.007 & 1.004 \\
\hline 8.01 & 1.014 & 1.008 & 1.007 & 1.005 & 1.002 \\
\hline
\end{tabular}

the corresponding average degree is 12

$$
\begin{aligned}
\lambda(x)= & 0.430034 x^{2}+0.237331 x^{12}+0.007979 x^{13} \\
& +0.119493 x^{47}+0.052153 x^{48} \\
& +0.079630 x^{161}+0.073380 x^{162} \\
\rho(x)= & 0.713788 x^{9}+0.122494 x^{10}+0.163718 x^{199} .
\end{aligned}
$$

Note that, in contrast to the examples above, the maximum node degrees in these graphs are much smaller. This makes them more practical for smaller values of $k$ than the codes giving rise to Table I.

\section{Implementations and Timings}

In this subsection, we report on some of the implementations of our codes. In all these examples a message consisting of 640000 packets was encoded into a vector of 1280000 packets, and each packet consisted of 256 bytes. The cascade consisted of three layers: a first layer consisting of $640 \mathrm{~K}$ nodes on the left, and $320 \mathrm{~K}$ nodes on the right, a second layer consisting of $320 \mathrm{~K}$ nodes on the left and $160 \mathrm{~K}$ nodes on the right, and a third layer consisting of $160 \mathrm{~K}$ nodes on the left and on the right. The edge distributions of the graphs used in the first and the second layer were the heavy tail/Poisson distribution discussed in Section IV. The edge distribution in the third layer was different, and used some of the analysis of Section V-A: the edge distribution on the left was a "double heavy tail" distribution, given by $\lambda(x):=\tilde{\lambda}\left(x^{2}\right)$, where $\tilde{\lambda}$ is the edge distribution function of the heavy tail distribution.

To chose an appropriate random bipartite graph $B$ with $E$ edges, $k$ nodes on the left, and $\beta k$ nodes on the right, the program started with a bipartite graph $B^{\prime}$ with $E$ nodes on both the 


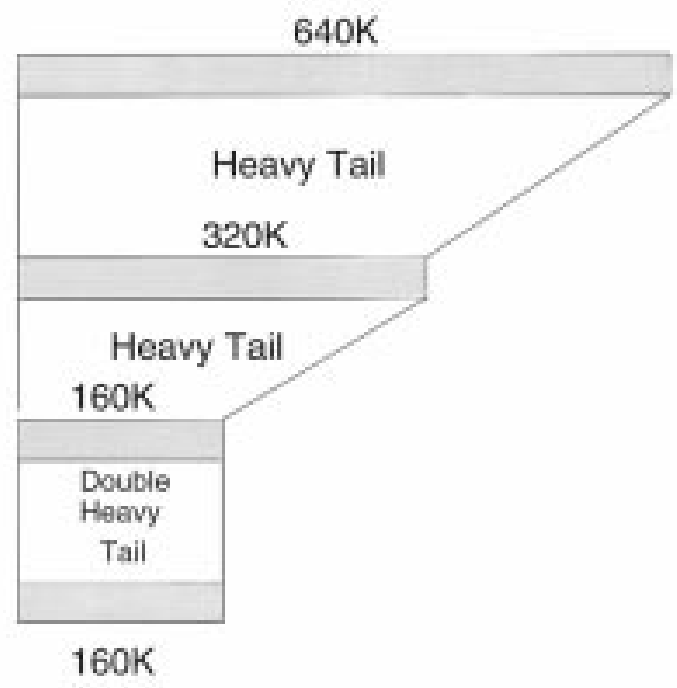

Fig. 7. Cascade for example.

left- and right-hand sides, with each node of $B^{\prime}$ representing an edge slot. Each node on the left-hand side of $B^{\prime}$ was associated with a node on the left side of $B$, so that the distribution of degrees is given by $\left(\lambda_{1}, \ldots, \lambda_{m}\right)$, and similarly for the right. The program then chooses a random matching (i.e., a random permutation) between the two sets of $E$ nodes on $B^{\prime}$. This induced a random bipartite graph on $B$ (perhaps with multiedges) in the obvious manner with the desired degree structure. In experiments, it turned out that the existence of multiedges is not a serious problem. This can be explained by the observation that one can analyze the process for random multigraphs instead of random graphs and that this analysis turns out to yield essentially the same results as the one carried out in Section III.

A schematic description of the code is given in Fig. 7. The average degree of the nodes in this graph was 8 . The decoding algorithm was executed 1000 times, each time with a different random loss pattern. Fig. 8 shows length overhead statistics: the horizontal axis represents $\mu$ and the vertical axis represents the percentage of times where $(1+\mu)$ times the length of the message was needed to completely recover the message, based on the 1000 trials. In compliance with the results of Section III, we see that the parameters are sharply concentrated around their mean value.

On a DEC-alpha machine with $300 \mathrm{MHz}$ and 64-Mbyte RAM the encoding took $0.58 \mathrm{CPU}$-seconds, and the decoding took $0.94 \mathrm{~s}$, on average. This corresponds to a throughput of roughly $280 \mathrm{Mbit} / \mathrm{s}$.

On a Pentium Pro at $200 \mathrm{MHz}$ and 64-Mbyte RAM, the encoding took $0.58 \mathrm{~s}$, while the decoding took $1.73 \mathrm{~s}$, on average. This corresponds to a throughput of roughly $150 \mathrm{Mbit} / \mathrm{s}$.

It should be noted that most of the time in our algorithm is spent in pointer chasing. The code used was a straightforward C implementation. Use of more sophisticated data types, and more intelligent prefetching strategies would probably speed up the code considerably.

\section{CONCLUSION}

We have introduced in this paper a class of error-correcting codes, based on a cascade of bipartite graphs. Although the idea of using sparse bipartite graphs for constructing codes is not new [6], [25], the construction of the graphs in each of the layers is novel. We obtained the construction by analyzing a simple decoding algorithm. The analysis used results asserting the sharp concentration of parameters in a discrete random process around their means. Using this, we established a simple condition that the degree sequences of the left- and right-hand sides of the bipartite graphs had to satisfy in order for the process to finish successfully. We designed a family of capacity-achieving codes on the erasure channel with linear time encoding and decoding algorithms. We should point out that our model of computation, as it stands, is that of a random-access machine with unit cost. However, our construction can be modified using prefetching strategies to yield linear time algorithms for random-access machines with logarithmic cost. The modification is quite similar to that given in [24].

\section{FURTHER DEVELOPMENTS}

The appearance of the first version of this paper as an extended abstractin [13] inspired new developments which we would like to briefly comment on in this section. First, the analysis of this paper was simplified in [9] by using proper martingale arguments. Nevertheless, since we feel that the approach outlined in this paper (in particular, Theorem 1) may have other applications, we opted for leaving the analysis in its original form. One of the main results of this paper is the fact that properly chosen irregular graphs perform a lot better than regular graphs, and that the only parameters that determine the asymptotic performance are the fractions of nodes of various degrees. This observation together with the new analysis were combined in [10] to study irregular low-density parity-check codes on the binary-symmetric channel, with simple hard-decision decoding algorithms going back to Gallager [6]. ${ }^{4}$ This paper appears to have been influential. First, the idea of using irregular codes was taken up and extended by other researchers (see, e.g., [14]). Second, the main "concentration theorem" of [10] was extended to a large class of channel models in a landmark paper by Richardson and Urbanke [22], which first appeared in 1998. Based on their approach, they developed the "density evolution" algorithm, a numerical procedure to approximate the threshold of noise below which the belief propagation algorithm ${ }^{5}$ is asymptotically successful. Several months later, their method was further extended in [21] in which sequences of codes were constructed for which the belief propagation algorithm had a performance extremely close to the Shannon capacity, beating turbo codes [2] by a wide margin for modest block lengths.

Another main result of this paper was to show that there are families of degree sequences such that the corresponding graphs asymptotically meet the capacity of the erasure channel (using our simple erasure-decoding algorithm). Another family of such degree sequences was exhibited in [23]. So far, these have been the only known capacity-achieving families of degree sequences, and another example of a communication channel for which capacity-achieving sequences exist for all rates is yet to be found.

\footnotetext{
${ }^{4} \mathrm{An}$ updated version of this paper appears in [12].

${ }^{5}$ Our erasure decoder turns out to be the belief propagation algorithm for the erasure channel [21]
} 


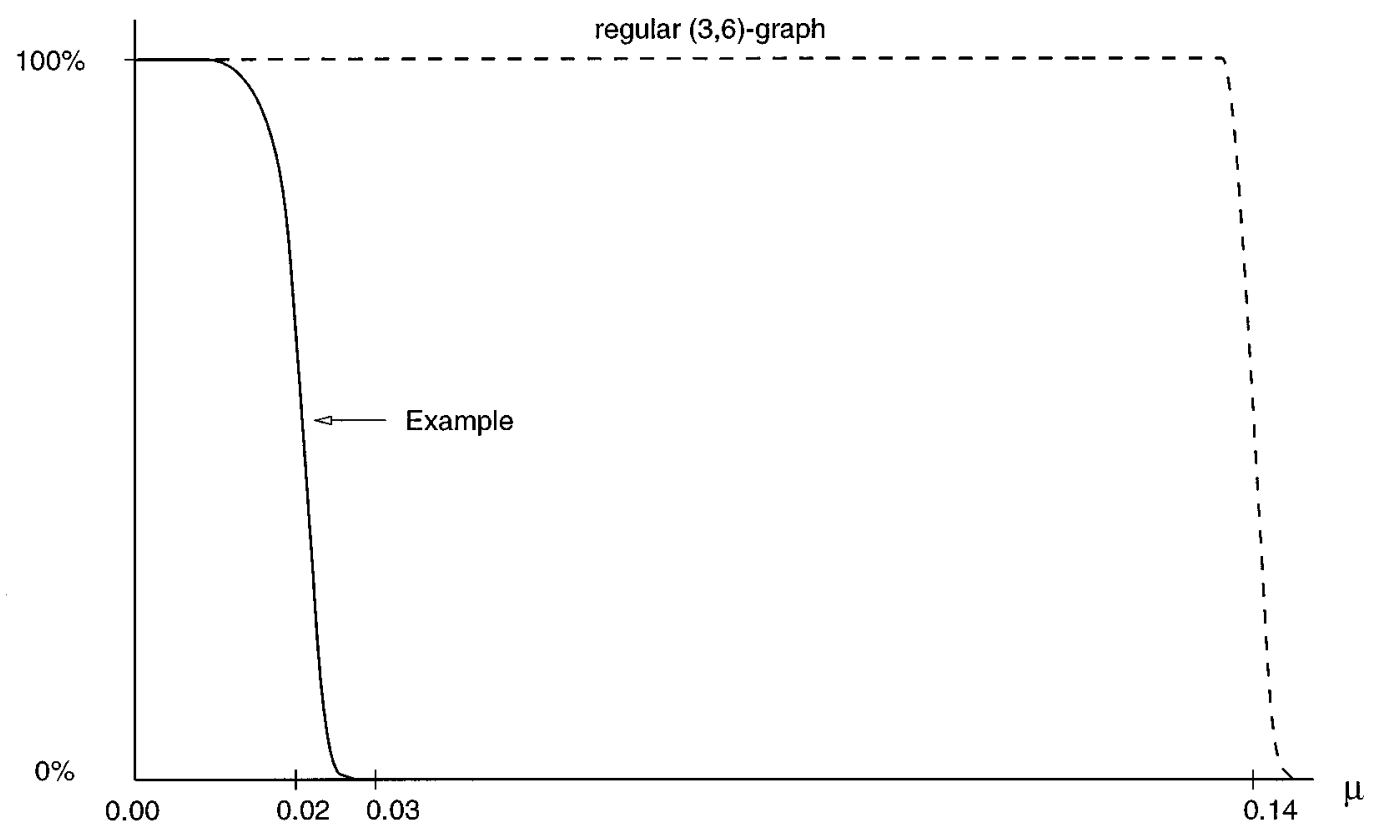

Fig. 8. Length overhead statistics.

APPENDIX A

\section{PROOF OF THEOREM 1}

Recall that a sequence of random variables $X_{0}, X_{1}, \ldots$ is called a martingale if

$$
E\left[X_{i} \mid X_{0}, \ldots, X_{i-1}\right]=X_{i-1}, \quad \text { for all } i \geq 1 .
$$

The sequence is called a submartingale (supermartingale) if $E\left[X_{i} \mid X_{0}, \ldots, X_{i-1}\right] \geq X_{i-1}\left(E\left[X_{i} \mid X_{0}, \ldots, X_{i-1}\right] \leq X_{i-1}\right)$.

For the proof of our concentration result we need the following well-known result, often called Azuma's inequality [26, Lemma 1].

Theorem 4: Let $X_{0}, X_{1}, \ldots$ be a supermartingale with respect to a sequence of $\sigma$-algebras $\mathcal{F}_{i}$ with $\mathcal{F}_{0}=\{\emptyset, \Omega\}$, and suppose that $X_{0}=0$ and $\left|X_{k+1}-X_{k}\right| \leq c$ for some constant $c$ and for $i \geq 0$. Then for all $\alpha>0$ we have

$$
\operatorname{Pr}\left(X_{k} \geq \alpha c\right) \leq \exp \left(-\alpha^{2} / 2 k\right)
$$

Proof of Theorem 1: We modify the proof in [26] slightly to obtain the error bounds asserted in the theorem. First, note that by a standard result in the theory of first-order differential equations, there is a unique solution in part a) of the theorem.

As in [26], we simplify the notation by considering $d=1$ and referring to $y^{(1, m)}, z_{1}$, and $f_{1}$ as $y, z$, and $f$, and so on. The proof for general $d$ is similar.

Let $w:=\left\lceil m^{2 / 3}\right\rceil$, and assume that $0 \leq t \leq m-w$. We first demonstrate concentration of $Y_{t+w}-Y_{t}$. Notice that the Lipschitz condition on $f$ and Condition ii) imply that for all $0 \leq k<w$

$$
\begin{aligned}
\boldsymbol{E}\left(Y_{t+k+1}-Y_{t+k} \mid H_{t+k}\right) & =f\left(\frac{t+k}{m}, \frac{Y_{t+k}}{m}\right) \\
& \leq f\left(\frac{t}{m}, \frac{Y_{t}}{m}\right)+\gamma \frac{2 k+1}{m}
\end{aligned}
$$

for some constant $\gamma$.
For fixed $t$, define the random variable

$$
X_{k}:=Y_{t+k}-Y_{t}-k f\left(t / m, Y_{t} / m\right)-\gamma k^{2} / m
$$

Note that

$$
X_{k+1}-X_{k}=Y_{t+k+1}-Y_{t+k}-f\left(\frac{t}{m}, \frac{Y_{t}}{m}\right)-\gamma \frac{2 k+1}{m} .
$$

This shows that the $X_{k}$ form a supermartingale with respect to $H_{t}, \ldots, H_{t+w}$, as

$$
0 \geq \boldsymbol{E}\left(X_{k+1}-X_{k} \mid H_{t+k}\right)=\boldsymbol{E}\left(X_{k+1} \mid H_{t+k}\right)-X_{k} .
$$

Furthermore, the above equality shows that $\left|X_{k+1}-X_{k}\right| \leq$ $C_{2}$ for some constant $C_{2}$. We can now apply the inequality of Theorem 4. As $X_{0}=0$, we obtain

$$
\operatorname{Pr}\left(X_{w} \geq \alpha C_{2}\right) \leq \exp \left(-\frac{\alpha^{2}}{2 w}\right)
$$

for any $0<\alpha$. (The parameter $\alpha$ will be chosen later.) The lower tail can be bounded in exactly the same way, using a submartingale. This gives for any constant $B$ (to be chosen later)

$$
\begin{aligned}
\operatorname{Pr}\left(\left|Y_{t+w}-Y_{t}-w f\left(\frac{t}{m}, \frac{Y_{t}}{m}\right)\right| \geq\right. & \left.(\gamma+B) \frac{w^{2}}{m}+\alpha C_{2}\right) \\
& \leq \exp \left(-\frac{\alpha^{2}}{2 w}\right) .
\end{aligned}
$$

Now let $k_{\ell}:=\ell w$, where $\ell=0,1, \ldots, \ell_{0}$ and

$$
\ell_{0}=\lfloor\min \{m / w, \sigma m / w\}\rfloor \text {. }
$$

Let

$$
T_{\ell}:=\frac{\left(\gamma w^{2}+\alpha m C_{2}\right)\left((1+B w / m)^{\ell}-1\right)}{B w} .
$$

We prove by induction on $\ell$ that

$$
\pi_{\ell}:=\operatorname{Pr}\left(\left|Y_{k_{\ell}}-z\left(k_{\ell} / m\right) m\right| \geq T_{\ell}\right) \leq \ell \exp \left(-\frac{\alpha^{2}}{2 w}\right) .
$$


The assertion is obvious for the induction starting at $\ell=0$, as $z(0)=\boldsymbol{E}\left(Y_{0}\right) / m$. Define

$$
\begin{aligned}
& A_{1}:=Y_{k_{\ell+1}}-Y_{k_{\ell}}-w f\left(k_{\ell} / m, Y_{k_{\ell}} / m\right) \\
& A_{2}:=Y_{k_{\ell}}-m z\left(k_{\ell} / m\right) \\
& A_{3}:=m z\left(k_{\ell+1} / m\right)-m z\left(k_{\ell} / m\right)-w f\left(k_{\ell} / m, Y_{k_{\ell}} / m\right) .
\end{aligned}
$$

Note that

$\left|Y_{k_{\ell+1}}-m z\left(k_{\ell+1} / m\right)\right|=\left|A_{1}+A_{2}-A_{3}\right| \leq\left|A_{1}\right|+\left|A_{2}\right|+\left|A_{3}\right|$.

The inductive hypothesis gives that $\left|A_{2}\right|<T_{\ell}$ with probability at least $1-\ell \exp \left(-\alpha^{2} / 2 w\right)$. Further, by (10), we have $\left|A_{1}\right|<$ $\gamma w^{2} / m+\alpha C_{2}$ with probability at least $1-\exp \left(-\alpha^{2} / 2 w\right)$. To bound $A_{3}$ we proceed as follows. By the mean value theorem we have that $z\left(k_{\ell+1} / m\right)-z\left(k_{\ell} / m\right)=w z^{\prime}(\xi) / m$, where $z^{\prime}$ is the derivative of $z$ and $\xi$ is some real number with $k_{\ell} / m \leq$ $\xi \leq k_{\ell+1} / m$. Note that $z$ satisfies the differential equation in (1), hence $z^{\prime}(\xi)=f(\xi, z(\xi))$, and by the Lipschitz condition on $f$ we obtain

$$
\left|A_{3}\right| \leq L w\left(\left|k_{\ell} / m-\xi\right|+\left|Y_{k_{\ell}} / m-z(\xi)\right|\right) .
$$

By the continuity of $z$ and the inductive hypothesis, we see that for suitable choice of the constant $B$ we have

$$
\left|A_{3}\right| \leq B\left(\frac{w^{2}}{m}+\frac{w T_{\ell}}{m}\right)
$$

for large enough $m$. Altogether we obtain

$$
\left|A_{1}\right|+\left|A_{2}\right|+\left|A_{3}\right| \leq T_{\ell+1}
$$

with probability at least

$1-\ell \exp \left(-\alpha^{2} / 2 w\right)-\exp \left(-\alpha^{2} / 2 w\right)=1-(\ell+1) \exp \left(-\alpha^{2} / 2 w\right)$.

Now we choose $\alpha=\sqrt{m}$. Then

$$
\begin{aligned}
T_{\ell} & \leq T_{m / w} \\
& \leq(\exp (B)-1)\left((\gamma+B) m^{4 / 3}+m^{3 / 2} C_{2}\right) /\left(B m^{2 / 3}\right) \\
& =O\left(m^{5 / 6}\right), \quad \text { for all } \ell .
\end{aligned}
$$

Hence, we see that (2) is satisfied at $t=k_{\ell}$ with probability at least $1-m^{2 / 3} \exp (-\sqrt[3]{m} / 2)$. Furthermore, as

$$
\left|Y_{t}-Y_{k_{\ell}}\right| \leq C^{\prime} m^{2 / 3}, \quad \text { for all } k_{\ell} \leq t \leq k_{\ell+1}
$$

we contend that $Y_{t}=m z(t / m)+O\left(m^{5 / 6}\right)$ for all $t$ in the specified range, with probability at least $1-m^{2 / 3} \exp (-\sqrt[3]{m} / 2)$.

We remark that one can have better choices for $\alpha$ and $w$ in the above proof which make the error terms smaller, at the expense of making the error probability slightly larger.

\section{APPENDIX B}

\section{PROOF OF PROPOSITION 1}

We will prove Proposition 1 in this appendix. We start with the substitution $x:=\exp \left(-\int_{0}^{\tau} d s / e(s)\right)$. This means that $d x / x=-d \tau / e(\tau)$, and this transforms for $i>1$ (3) into

$$
r_{i}^{\prime}(x)=i\left(-r_{i+1}(x)+r_{i}(x)\right) \frac{a(x)-1}{x}
$$

where prime stands for derivative with respect to the variable $x$, and $a(x)$ is the average degree of the graph at time $x$. Note that $a(x)$ equals $\sum i \ell_{i}(x) / e(x)$, which in terms of the function $\lambda(x)$ can be written as $1+x \lambda^{\prime}(x) / \lambda(x)$. Hence, we obtain for $i>1$

$$
r_{i}^{\prime}(x)=i\left(-r_{i+1}(x)+r_{i}(x)\right) \frac{\lambda^{\prime}(x)}{\lambda(x)} .
$$

As is verified easily, the explicit solution is given by

$$
r_{i}(x)=\lambda(x)^{i}\left(-i \int_{0}^{x} r_{i+1}(y) \lambda(y)^{-i} \frac{\lambda^{\prime}(y)}{\lambda(y)} d y+c_{i}\right)
$$

for some constants $c_{i}$ to be determined from the initial conditions for $r_{i}$. These equations can be solved recursively, starting with the highest nonzero $r_{i}$, say $r_{\mu}$. In this case, we have $r_{\mu}^{\prime}(x)=\mu r_{\mu}(x) \lambda^{\prime}(x) / \lambda(x)$, which gives $r_{\mu}(x)=c_{\mu} \lambda(x)^{\mu}$ for some constant $c_{\mu}$. Using induction, it is then easy to prove that

$$
r_{i}(x)=\sum_{j \geq i}(-1)^{i+j}\left(\begin{array}{c}
j-1 \\
i-1
\end{array}\right) c_{j} \lambda(x)^{j} .
$$

Further, since $\lambda(1)=1$, one verifies by induction that

$$
c_{i}=\sum_{j \geq i}\left(\begin{array}{l}
j-1 \\
i-1
\end{array}\right) r_{j}(1) .
$$

Plugging (5) into the last equation we see that

$$
c_{i}=\sum_{m \geq i}\left(\begin{array}{c}
m-1 \\
i-1
\end{array}\right) \rho_{m} \delta^{i} .
$$

(Use $\left(\begin{array}{c}m-1 \\ j-1\end{array}\right)\left(\begin{array}{c}j-1 \\ i-1\end{array}\right)=\left(\begin{array}{c}m-1 \\ i-1\end{array}\right)\left(\begin{array}{c}m-j \\ j-i\end{array}\right)$.) Hence, we obtain for $i>1$ from (12)

$$
r_{i}(x)=\sum_{m \geq j \geq i}(-1)^{i+j}\left(\begin{array}{c}
j-1 \\
i-1
\end{array}\right)\left(\begin{array}{c}
m-1 \\
j-1
\end{array}\right) \rho_{m}(\delta \lambda(x))^{j} .
$$

To obtain the formula for $r_{1}(x)$, we note that $r_{1}(x)=e(x)-$ $\sum_{i>1} r_{i}(x)$. The sum of the right-hand side of (13) over all $i \geq$ 1 equals

$\sum_{m \geq j}(-1)^{j-1}\left(\begin{array}{c}m-1 \\ j-1\end{array}\right) \rho_{m}(\delta \lambda(x))^{j} \sum_{i \leq j}(-1)^{i-1}\left(\begin{array}{l}j-1 \\ i-1\end{array}\right)$

(The inner sum equals 1 if $j=1$, and is zero otherwise.) Hence, we have

$$
\begin{aligned}
r_{1}(x)= & e(x)-\delta \lambda(x)+\delta \lambda(x) \sum_{m} \rho_{m} \sum_{j \leq m}(-1)^{j-1} \\
& \cdot\left(\begin{array}{c}
m-1 \\
j-1
\end{array}\right)(\delta \lambda(x))^{j-1} \\
= & x \delta \lambda(x)-\delta \lambda(x)+\delta \lambda(x) \sum_{m} \rho_{m}(1-\delta \lambda(x))^{m-1} \\
= & \delta \lambda(x)[x-1+\rho(1-\delta \lambda(x))] .
\end{aligned}
$$

This completes the proof.

\section{APPENDIX C}

\section{PROOF OF LEMMA 4}

Again, we begin with the initial random graph $B$, with $k$ left nodes and $\beta k$ right nodes, and continue to work with the generating functions $\lambda(x)$ and $\rho(x)$ from Section III. Suppose that each node on the right is removed with probability $\delta^{\prime}$, while nodes on the left are removed with probability $\delta$. The 
new process can now be studied as a process with erasures on the left only, which runs on the subgraph $\tilde{B}$ of the initial consisting of the $\left(1-\delta^{\prime}\right) \beta n$ undeleted nodes on the right. Let $\tilde{\lambda}_{i}$ be the fraction of edges of degree $i$ in $\tilde{B}$ with respect to the total number of edges in $\tilde{B}$. Define $\tilde{\rho}$ similarly. Obviously, $\tilde{\rho}_{i}=\rho_{i}$, as the number of edges of degree $i$ and the number of total edges in $\tilde{B}$ are a $\left(1-\delta^{\prime}\right)$-factor of those of $B$. As for $\tilde{\lambda}_{i}$, it is easily seen that

$$
\tilde{\lambda}_{i}=\sum_{j \geq i} \lambda_{j}\left(\begin{array}{c}
j-1 \\
i-1
\end{array}\right)\left(1-\delta^{\prime}\right)^{i}\left(\delta^{\prime}\right)^{j-i} .
$$

This is done as follows: an edge of degree $j$ is with probability $1-\delta^{\prime}$ connected to an undeleted node on the right. The probability that $j-i$ of the remaining $j-1$ edges is connected to one of the deleted nodes on the right is exactly a $\left(1-\delta^{\prime}\right)$-fraction of the above sum.

The above formula shows that $\tilde{\lambda}(x)=\lambda\left(\delta^{\prime}+\left(1-\delta^{\prime}\right) x\right)$. Invoking Theorem 1 we see that the expected number of edges of right degree one at time $x$ (with respect to the total number of edges in $\tilde{B}$ ) equals

$$
\delta \lambda\left(\delta^{\prime}+\left(1-\delta^{\prime}\right) x\right)\left[x-1+\rho\left(1-\delta \lambda\left(\delta^{\prime}+\left(1-\delta^{\prime}\right) x\right)\right)\right] .
$$

Since the number of edges in $\tilde{B}$ is $\left(1-\delta^{\prime}\right)$ times the number of edges in $B$, the assertion on $r_{1}(x)$ follows.

To prove the second part of the proposition, we retain the notation established earlier, e.g., $e(x)$ is the fraction of the original edges remaining at $x$. Let $E$ be the number of edges in the original graph, $N$ be the number of left nodes in the original graph, and thus the average left node degree is $a_{\ell}=E / N$. We define $b(x)$ to be the average node degree among nodes on the left that have at least one edge at $x$.

We define $f_{i}$ to be the fraction of left nodes of degree $i$ in the original graph, and thus $f_{i}=a_{\ell} \cdot \lambda_{i} / i$. We define $f(x)$ to be the expected fraction of original left nodes still not recovered at $x$. We define $\tilde{f}$ to be the fraction of left nodes that have all their neighbors among the original $\delta^{\prime}$ fraction of missing right nodes. We define $\hat{f}(x)$ to be the expected fraction of left nodes that have at least one neighbor not among the original $\delta^{\prime}$ fraction of missing right nodes and that are still not recovered at $x$.

One can verify that $f(x)=\delta \tilde{f}+\hat{f}(x)$, and that $\tilde{f}=$ $\sum_{i} f_{i}\left(\delta^{\prime}\right)^{i}$. Thus, our goal is to deduce a closed-form expression for $\hat{f}(x)$. The number of unrecovered left nodes with at least one neighbor at $x$ is equal to the number of edges remaining at $x$ divided by $b(x)$. The number of edges at $x$ is $e(x) E$, and thus

$$
\hat{f}(x)=\frac{e(x) E}{b(x) N}=a_{\ell} \cdot e(x) / b(x) .
$$

We now turn to $b(x)$. It can be verified that

$$
b(x)=\frac{e(x)}{\int_{0}^{x} e(y) / y d y} .
$$

From this it follows that $\hat{f}(x)=a_{\ell} \cdot \int_{0}^{x} e(y) / y d y$. Recall that $e(y)=\delta\left(1-\delta^{\prime}\right) y \lambda\left(\delta^{\prime}+\left(1-\delta^{\prime}\right) y\right)$, and thus

$$
e(y) / y=\delta\left(1-\delta^{\prime}\right) \lambda\left(\delta^{\prime}+\left(1-\delta^{\prime}\right) y\right) .
$$

Further, recall that $\lambda(z)=\sum_{i} \lambda_{i} z^{i-1}$. Thus

$$
\int_{0}^{x} \lambda\left(\delta^{\prime}+\left(1-\delta^{\prime}\right) y\right) d y=\left.\sum_{i} \frac{\lambda_{i}}{i} \cdot \frac{\left(\delta^{\prime}+\left(1-\delta^{\prime}\right) y\right)^{i}}{1-\delta^{\prime}}\right|_{0} ^{x} .
$$

Thus

$$
\begin{aligned}
\int_{y}^{x} e(y) / y d y & =\left.\delta \sum_{i} \frac{\lambda_{i}}{i} \cdot\left(\delta^{\prime}+\left(1-\delta^{\prime}\right) y\right)^{i}\right|_{0} ^{x} \\
& =\left.\frac{\delta}{a_{\ell}} \sum_{i} f_{i} \cdot\left(\delta^{\prime}+\left(1-\delta^{\prime}\right) y\right)^{i}\right|_{0} ^{x} .
\end{aligned}
$$

This implies

$$
\hat{f}(x)=\delta\left[\sum_{i} f_{i} \cdot\left(\delta^{\prime}+\left(1-\delta^{\prime}\right) x\right)^{i}-\tilde{f}\right] .
$$

Finally

$$
f(x)=\hat{f}(x)+\delta \tilde{f}=\delta \sum_{i} f_{i} \cdot\left(\delta^{\prime}+\left(1-\delta^{\prime}\right) x\right)^{i} .
$$

By using Theorem 1, this shows that the fraction of nodes unrecovered at time $x$ is, up to small order terms, equal to

$$
\delta a_{\ell} \cdot \int_{0}^{\left(\delta^{\prime}+\left(1-\delta^{\prime}\right) x\right)} \lambda(y) d y
$$

and completes the proof.

\section{REFERENCES}

[1] N. Alon and M. Luby, "A linear time erasure-resilient code with nearly optimal recovery,” IEEE Trans. Inform. Theory, vol. 42, pp. 1732-1736, Nov. 1996.

[2] C. Berrou, A. Glavieux, and P. Thitimajshima, "Near Shannon limit error-correcting coding and decoding," in Proc. Int. Conf. Communication (ICC'93), Geneva, Switzerland, May 1993, pp. 1064-1070.

[3] R. E. Blahut, Theory and Practice of Error Control Codes. Reading, MA: Addison-Wesley, 1983.

[4] P. Elias, "Coding for two noisy channels," in Information Theory, 3rd London Symp., 1955, pp. 61-76.

[5] A. Frieze and S. Suen, "Analysis of two simple heuristics on a random instance of $k$-SAT," J. Algorithms, vol. 20, pp. 312-355, 1996.

[6] R. G. Gallager, Low Density Parity-Check Codes. Cambridge, MA: MIT Press, 1963.

[7] R. Karp and M. Sipser, "Maximum matchings in sparse random graphs," in Proc. 22nd FOCS, 1981, pp. 364-375.

[8] T. G. Kurtz, "Approximation of population processes," in CBMS-NSF Regional Conf. Series in Applied Math., SIAM, 1981.

[9] M. Luby, M. Mitzenmacher, and M. A. Shokrollahi, "Analysis of random processes via and-or tree evaluation," in Proc. 9th Annu. ACM-SIAM Symp. Discrete Algorithms, 1998, pp. 364-373.

[10] M. Luby, M. Mitzenmacher, M. A. Shokrollahi, and D. Spielman, "Analysis of low density codes and improved designs using irregular graphs," in Proc. 30th Annu. ACM Symp. Theory of Computing, 1998, pp. 249-258.

[11] — "Improved low-density parity-check codes using irregular graphs and belief propagation," in Proc. 1998 IEEE Int. Symp. Information Theory, 1998, p. 117.

[12] - "Analysis of low density codes and improved designs using irregular graphs," IEEE Trans. Inform. Theory, vol. 47, pp. 671-686, Feb. 2000.

[13] M. Luby, M. Mitzenmacher, M. A. Shokrollahi, D. Spielman, and V. Stemann, "Practical loss-resilient codes," in Proc. 29th Annu. ACM Symp. Theory of Computing, 1997, pp. 150-159.

[14] D. MacKay, S. Wilson, and M. Davey, "Comparison of constructions of irregular Gallager codes," IEEE Trans. Commun., vol. 47, no. 10, pp. 1449-1454, Oct. 1999.

[15] D. J. C. MacKay and R. M. Neal, "Near Shannon limit performance of low density parity check codes," Electron. Lett., vol. 32, no. 18, pp. 1645-1646, 1996. 
[16] F. J. MacWilliams and N. J. A. Sloane, The Theory of Error-Correcting Codes. Amsterdam, The Netherlands: North-Holland, 1988.

[17] M. Mitzenmacher, "The power of two choices in randomized load balancing," Ph.D. dissertation, Univ. Calif, Berkeley, 1996.

[18] _ - "Tight thresholds for the pure literal rule," DEC/SRC, Tech. Rep. 1997-011, 1997.

[19] R. Motwani and P. Raghavan, Randomized Algorithms. Cambridge, U.K.: Cambridge Univ. Press, 1995.

[20] B. Pittel, J. Spencer, and N. Wormald, "Sudden emergence of a giant $k$-core in a random graph," J. Comb. Theory, Ser. B, vol. 67, pp. 111-151, 1996.

[21] T. Richardson, M. A. Shokrollahi, and R. Urbanke, "Design of capacityapproaching irregular low-density parity-check codes," IEEE Trans. Inform. Theory, vol. 47, pp. 619-637, Feb. 2000.
[22] T. Richardson and R. Urbanke, "The capacity of low-density parity check codes under message-passing decoding," IEEE Trans. Inform. Theory, vol. 47, pp. 599-618, Feb. 2000.

[23] M. A. Shokrollahi, "New sequences of linear time erasure codes approaching the channel capacity," in Proc. 13th Int. Symp. Applied Algebra, Algebraic Algorithms, and Error-Correcting Codes (Lecture Notes in Computer Science), M. Fossorier, H. Imai, S. Lin, and A. Poli, Eds. Berlin, Germany: Springer-Verlag, 1999, pp. 65-76.

[24] M. Sipser and D. Spielman, "Expander codes," IEEE Trans. Inform. Theory, vol. 42, pp. 1710-1722, Nov. 1996.

[25] D. Spielman, "Linear-time encodable and decodable error-correcting codes," IEEE Trans. Inform. Theory, vol. 42, pp. 1723-1731, Nov. 1996.

[26] N. C. Wormald, "Differential equations for random processes and random graphs," Ann. Appl. Probab., vol. 5, pp. 1217-1235, 1995. 